\title{
Maxmaxflow and Counting Subgraphs
}

\author{
Bill Jackson \\ School of Mathematical Sciences \\ Queen Mary University of London \\ Mile End Road \\ London E1 4NS, England \\ B . JACKSON@QMUL . AC . UK \\ Alan D. Sokal* \\ Department of Physics \\ New York University \\ 4 Washington Place \\ New York, NY 10003 USA \\ SOKAL@NYU.EDU
}

Submitted: Sep 28, 2009; Accepted: Jun 28, 2010; Published: Jul 10, 2010

Mathematics Subject Classification: 05C99 (Primary);

05C15, 05C30, 05C35, 05C40, 82B20, 90B10 (Secondary)

\begin{abstract}
We introduce a new graph invariant $\Lambda(G)$ that we call maxmaxflow, and put it in the context of some other well-known graph invariants, notably maximum degree and its relatives. We prove the equivalence of two "dual" definitions of maxmaxflow: one in terms of flows, the other in terms of cocycle bases. We then show how to bound the total number (or more generally, total weight) of various classes of subgraphs of $G$ in terms of either maximum degree or maxmaxflow. Our results are motivated by a conjecture that the modulus of the roots of the chromatic polynomial of $G$ can be bounded above by a function of $\Lambda(G)$.
\end{abstract}

Key Words: Graph, subgraph, flow, cocycle, maxmaxflow, maximum degree, secondlargest degree, degeneracy number, chromatic polynomial.

${ }^{*}$ Also at Department of Mathematics, University College London, London WC1E 6BT, England.

THE ELECTRONIC JOURNAL OF COMBINATORICS 17 (2010), \#R99 


\section{Contents}

1 Introduction $\quad 2$

2 Maxmaxflow $\quad 4$

3 Cocycle Bases and Maxmaxflow $\quad 7$

4 Some Preliminaries 12

4.1 Pointwise bounds vs. generating-function bounds . . . . . . . . . . . . 12

4.2 Convex hulls in graphs . . . . . . . . . . . . . . . . . . . . 12

5 Counting Walks and Paths 13

6 Counting Trees and Forests 18

6.1 The classes $\mathcal{T}_{m}(X)$ and $\mathcal{F}_{m}(X, Y) \ldots \ldots \ldots \ldots$

6.2 The class $\mathcal{H}_{m}(X) \ldots \ldots \ldots \ldots \ldots$. . . . . . . . . . . . . . . 22

7 Counting Connected Subgraphs (and Related Objects) 24

8 Counting Blocks, Block Paths, Block Trees and Block Forests $\quad 29$

8.1 The classes $\mathcal{B T}_{m}(X), \mathcal{B F}_{m}(X, Y)$ and $\mathcal{B F}_{m}^{*}(X, Y) \ldots \ldots . . . . .30$

8.2 The class $\mathcal{B}_{m}(X) \ldots \ldots \ldots \ldots$. . . . . . . . . . . . . . . 42

\section{Introduction}

An elementary result on graph colouring is that the chromatic number $\chi(G)$ of a graph $G$ is at most one more than the maximum degree $\Delta(G)$. A much deeper result is that the modulus of the roots (real or complex) of the chromatic polynomial of $G$ can be bounded above by a linear function of $\Delta(G)$, see [14]. Indeed, a similar bound holds when the maximum degree $\Delta(G)$ is replaced by the second-largest degree $\Delta_{2}(G)$, although the currently available proof of this fact [14, Corollary 6.4] is somewhat ad hoc. ${ }^{1}$

One obvious drawback in all these results is that we can make the maximum degree and second-largest degree arbitrarily large by gluing together many copies of $G$ in a treelike fashion at cut vertices, without changing the chromatic number or the chromatic roots. Another (related) drawback is that there is no obvious way to extend these results from graphs to matroids and thereby to obtain dual results for nowhere-zero flows and the roots of flow polynomials.

The purpose of this paper is to introduce a new graph invariant $\Lambda(G)$ that we call maxmaxflow, which we conjecture will give a more natural upper bound on chromatic

\footnotetext{
${ }^{1}$ Note that it is not possible to go farther and obtain a bound in terms of the third-largest degree $\Delta_{3}$, as the chromatic roots of the generalized theta graphs $\Theta^{(s, p)}$ - which have $\Delta=\Delta_{2}=p$ but $\Delta_{3}=2-$ are dense in the whole complex plane with the possible exception of the disc $|q-1|<1[15$, Theorems $1.1-1.4]$.
} 
roots. The maxmaxflow $\Lambda(G)$ is defined as the maximum, over all pairs of distinct vertices $x, y$ of $G$, of the maximum number of pairwise edge-disjoint $x y$-paths. It is easy to see that $\Lambda(G)$ is less than or equal to $\Delta_{2}(G)$, and that the maxmaxflow of any graph is equal to the largest maxmaxflow in its blocks (maximal non-separable subgraphs). We will show that $\Lambda(G)$ can equivalently be defined in terms of the bases of the cocycle space of $G$, so that the definition of maxmaxflow can be extended to binary matroids. We will furthermore see that $\Lambda(G)$ is at least as large as the degeneracy number $D(G)$ of $G$, so that we have $\chi(G) \leqslant D(G)+1 \leqslant \Lambda(G)+1$. We conjecture that $\Lambda(G)$ can also be used to give a bound on the chromatic roots of $G$ :

Conjecture 1.1 There exist universal constants $C(\Lambda)<\infty$ such that all the chromatic roots (real or complex) of all loopless graphs of maxmaxflow $\Lambda$ lie in the disc $|q| \leqslant C(\Lambda)$. Indeed, we conjecture that $C(\Lambda)$ can be taken to be linear in $\Lambda$.

This conjecture first appeared in [14, Section 7] and was inspired by a suggestion of Shrock and Tsai $[12,13]$. It has very recently been proven for series-parallel graphs by Royle and Sokal [11].

An important step in the proof [14] that the chromatic roots of $G$ can be bounded in terms of $\Delta(G)$ is obtaining an exponential upper bound in terms of $\Delta(G)$ for the number of connected $m$-edge subgraphs containing a fixed vertex of $G$. The approach in [14] is to decompose a spanning subgraph of $G$ into its connected components and to treat these components as a "polymer gas". The desired bound on chromatic roots then follows from standard bounds on the zeros of a polymer-gas partition function, once one has the exponential bound on the number of connected $m$-edge subgraphs containing a specified vertex.

Unfortunately, the number of connected $m$-edge subgraphs containing a fixed vertex cannot be bounded in terms of $\Lambda(G)$. This can easily be seen by taking $G$ to be large star: we have $\Lambda(G)=1$ and yet there is no bound on the number of connected $m$-edge subgraphs containing the central vertex.

Since both the chromatic polynomial and maxmaxflow "factorize over blocks", it is natural to try to prove Conjecture 1.1 by modifying the arguments of [14] to decompose a spanning subgraph of $G$ into its blocks rather than its connected components. The main result of this paper, Corollary 8.5, is a first step in this direction. It shows - a result that some readers may find surprising - that the number of non-separable $m$-edge subgraphs containing a fixed edge of $G$ satisfies an exponential upper bound in terms of $\Lambda(G)$. This will be good enough to prove Conjecture 1.1 provided that other difficulties (such as controlling the interaction between blocks) can be overcome.

Irrespective of the potential application to bounding chromatic roots, we think that maxmaxflow is a natural graph invariant that deserves further study and that bounds on the number of subgraphs of various kinds in terms of $\Delta(G)$ or $\Lambda(G)$ are of independent interest. $^{2}$

\footnotetext{
${ }^{2}$ See Section 2 below for references to scattered earlier work concerning maxmaxflow.
} 
The plan of this paper is as follows: In Section 2 we introduce maxmaxflow and put it in the context of some other well-known graph invariants (notably maximum degree and its relatives and degeneracy number). In Section 3 we analyze cocycle bases and prove the equivalence of the two definitions of maxmaxflow; an important role in this proof is played by Gomory-Hu trees [4]. The remainder of the paper is devoted to bounding the total number (or more generally, total weight) of various classes of subgraphs in terms of either maximum degree or maxmaxflow. Our basic approach is to start with a bound (sometimes a known one, sometimes a new one) in terms of maximum degree, and then see whether we can find a similar bound in terms of maxmaxflow. After some brief preliminaries (Section 4), we analyze walks and paths (Section 5) and then trees and forests (Section 6). In Section 7 we consider connected subgraphs and in Section 8 we consider non-separable subgraphs. Roughly speaking, the (more difficult) proofs in the later sections are constructed by adapting ideas from the (easier) proofs in the earlier sections. We hope that, by organizing the paper in terms of gradually increasing complexity of proof, we have helped to reduce the mental burden on the reader.

\section{Maxmaxflow}

Let $G$ be a finite undirected graph with vertex set $V(G)$ and edge set $E(G)$; in this paper all graphs are assumed to be loopless, but multiple edges are allowed unless explicitly specified otherwise. We shall say that $G$ is simple if it has no multiple edges. Let $\Delta(G)=\max _{x \in V(G)} d_{G}(x)$ be the maximum degree of $G$, and more generally let $\Delta_{k}(G)$ be the $k$ th largest degree of $G$ :

$$
\Delta_{k}(G)=\min _{x_{1}, \ldots, x_{k-1} \in V(G)} \max _{x \in V(G) \backslash\left\{x_{1}, \ldots, x_{k-1}\right\}} d_{G}(x) .
$$

We trivially have

$$
\delta(G) \equiv \Delta_{n}(G) \leqslant \cdots \leqslant \Delta_{3}(G) \leqslant \Delta_{2}(G) \leqslant \Delta_{1}(G) \equiv \Delta(G)
$$

where $n=|V(G)|$. A special role will be played in this paper by the second-largest degree, $\Delta_{2}(G)$.

For $x, y \in V(G)$ with $x \neq y$, the maximum flow from $x$ to $y$ in $G$ is

$$
\begin{aligned}
\lambda_{G}(x, y) & =\max \# \text { of edge-disjoint paths from } x \text { to } y \\
& =\min \# \text { of edges separating } x \text { from } y
\end{aligned}
$$

We then define the maxmaxflow of $G$

$$
\Lambda(G)=\max _{\substack{x, y \in V(G) \\ x \neq y}} \lambda_{G}(x, y) .
$$

[Note the contrast with the edge-connectivity, which is the minimum of $\lambda_{G}(x, y)$ over $x \neq y$.] Clearly $\lambda_{G}(x, y) \leqslant \min \left[d_{G}(x), d_{G}(y)\right]$, so that

$$
\Lambda(G) \leqslant \Delta_{2}(G)
$$


We will show later (Proposition 3.10) that $\Lambda(G) \geqslant \Delta_{n-1}(G)$. Note that several cases can arise:

(a) $\Lambda(G)=\Delta_{2}(G)=\Delta(G)$. Indeed, in any regular graph one has $\Lambda(G)=\Delta_{i}(G)$ for all $i(1 \leqslant i \leqslant n)$.

(b) $\Lambda(G)=\Delta_{2}(G) \ll \Delta(G)$. This occurs, for example, in stars $K_{1, r}$ and wheels $K_{1}+C_{r}$.

(c) More generally, one can have $\Lambda(G)=\Delta_{j+1}(G) \ll \Delta_{j}(G)$ for any fixed integer $j$. Moreover, such examples can be taken to be $k$-connected for arbitrarily large $k .^{3}$

Note also that maxmaxflow has a naturalness property that maximum degree and $k$ thlargest degree lack, namely, it "trivializes over blocks": $\Lambda(G)=\max _{1 \leqslant i \leqslant b} \Lambda\left(G_{i}\right)$ where $G_{1}, \ldots, G_{b}$ are the blocks of $G$ (Proposition 3.11).

Maxmaxflow appears to have been considered sporadically in the graph-theoretic literature. Bollobás [2, section I.5] addresses some extremal problems involving maxmaxflow in simple graphs (he uses the term "maximum local edge-connectivity" and denotes it $\bar{\lambda}(G))$; see likewise Mader [10, section IV]. In particular, Mader [9] has shown that whenever an $n$-vertex graph has more than $k(n-1) / 2$ edges, it has maxmaxflow at least $k$, but that for every $n \geqslant k \geqslant 2$ there exists an $n$-vertex graph with exactly $\lfloor k(n-1) / 2\rfloor$ edges and maxmaxflow $k-1 .^{4}$

An apparently very different quantity can be defined via cocycle bases. For $X, Y$ disjoint subsets of $V(G)$, let $E(X, Y)$ denote the set of edges in $G$ between $X$ and $Y$. A cocycle of $G$ is a set $E\left(X, X^{c}\right)$ where $X \subseteq V(G)$ and $X^{c} \equiv V(G) \backslash X$. It is well-known that the cocycles of $G$ form a vector space over $\mathrm{GF}(2)$ with respect to symmetric difference; this is called the cocycle space of $G$. Let $\widetilde{\Lambda}(G)$ be the minmax cardinality of the cocycles in a basis, i.e.

$$
\widetilde{\Lambda}(G)=\min _{\mathcal{B}} \max _{C \in \mathcal{B}}|C|
$$

where the min runs over all bases $\mathcal{B}$ of the cocycle space of $G$. Since one special class of cocycle bases consists of taking the stars $C(x)=E\left(\{x\},\{x\}^{c}\right)$ for all but one of the vertices in each component of $G$, we clearly have

$$
\widetilde{\Lambda}(G) \leqslant \Delta_{2}(G)
$$

\footnotetext{
${ }^{3}$ Proof. For $1 \leqslant i \leqslant j$, let $H_{i}$ be a $k$-connected graph with one vertex $v_{i}$ of degree $\Delta \gg k$ and all other vertices of degree $k$. [Such graphs can be constructed by taking a $(k-1)$-connected $(k-1)$-regular graph with a large number $\Delta$ of vertices and adding a new vertex $v_{i}$ adjacent to every other vertex.] Construct $G$ from the disjoint union of $H_{1}, H_{2}, \ldots, H_{j}$ by adding $k$ edges between each pair $H_{i}-v_{i}$ and $H_{i+1}-v_{i+1}(1 \leqslant i \leqslant j-1)$ in such a way that the set of edges of $G$ which do not belong to any $H_{i}$ are independent. [This can be done as long as $\left|V\left(H_{i}\right)\right| \geqslant 2 k+1$.] Then $G$ is $k$-connected and satisfies $\Lambda(G)=\Delta_{j+1}(G)=k+1$. [Since all pairs of vertices of $G$ with degrees greater than $k+1$ are of the form $v_{s}, v_{t}$ with $s \neq t$, and hence are separated by a set of $k$ edges, we have $\Lambda(G) \leqslant k+1$. On the other hand, if we choose two vertices $x, y \in H_{1}$ that are both adjacent to $H_{2}$, we can find $k$ edge-disjoint $x y$-paths in $H_{1}$ (since $H_{1}$ is $k$-connected) and an extra $x y$-path passing through $H_{2}$.] But $\Delta_{j}(G)=\Delta$.

${ }^{4}$ For $k=2,3$ this is easy. For $k=4$ it was proven earlier by Bollobás [1], and for $k=5,6$ by Leonard $[6,7]$.
} 
The relationship, if any, between maxmaxflow and cocycle bases is perhaps not obvious at first sight. But we shall prove (Corollary 3.9) that

$$
\Lambda(G)=\widetilde{\Lambda}(G) .
$$

The two definitions thus give dual approaches to the same quantity.

Finally, define the degeneracy number $D(G)=\max _{H \subseteq G} \delta(H)$, where the max runs over all subgraphs $H$ of $G$, and $\delta(H)$ is the minimum degree of $H$. It is easy to see that

$$
D(G) \leqslant \Delta_{2}(G)
$$

[if $H$ has at least two vertices, then $\delta(H) \leqslant \Delta_{2}(G)$; otherwise $\delta(H)=0$ ]. We shall in fact show (Proposition 3.10) that

$$
D(G) \leqslant \Lambda(G) .
$$

In summary, therefore, we have

$$
D(G) \leqslant \Lambda(G)=\widetilde{\Lambda}(G) \leqslant \Delta_{2}(G) \leqslant \Delta(G) .
$$

The natural setting for the results of this paper is, in fact, that of a finite undirected loopless (multi)graph $G$ equipped with nonnegative real edge weights $\mathbf{w}=\left\{w_{e}\right\}_{e \in E(G)}$. Indeed, all of the aforementioned invariants have natural generalizations to this context. Define first the weighted degree of a vertex,

$$
d_{G}(x, \mathbf{w})=\sum_{e \ni x} w_{e} .
$$

We then set

$$
\begin{aligned}
\Delta(G, \mathbf{w}) & =\max _{x \in V(G)} d_{G}(x, \mathbf{w}) \\
\Delta_{k}(G, \mathbf{w}) & =\min _{x_{1}, \ldots, x_{k-1} \in V(G)} \max _{x \in V(G) \backslash\left\{x_{1}, \ldots, x_{k-1}\right\}} d_{G}(x, \mathbf{w}) \\
\delta(G, \mathbf{w}) & =\min _{x \in V(G)} d_{G}(x, \mathbf{w}) \\
\widetilde{\Lambda}(G, \mathbf{w}) & =\min _{\mathcal{B}} \max _{C \in \mathcal{B}} \sum_{e \in C} w_{e} \\
D(G, \mathbf{w}) & =\max _{H \subseteq G} \delta\left(H,\left.\mathbf{w}\right|_{H}\right)
\end{aligned}
$$

Likewise, max-flow quantities are naturally defined when the $\left\{w_{e}\right\}$ are interpreted as edge capacities:

$$
\begin{aligned}
\lambda_{G}(x, y ; \mathbf{w}) & =\max \text { flow from } x \text { to } y \text { with edge capacities } \mathbf{w} \\
& =\text { min cut between } x \text { and } y \text { with edge capacities } \mathbf{w}
\end{aligned}
$$


and thence

$$
\Lambda(G, \mathbf{w})=\max _{\substack{x, y \in V(G) \\ x \neq y}} \lambda_{G}(x, y ; \mathbf{w}) .
$$

In this generality we shall prove

$$
D(G, \mathbf{w}) \leqslant \Lambda(G, \mathbf{w})=\widetilde{\Lambda}(G, \mathbf{w}) \leqslant \Delta_{2}(G, \mathbf{w}) \leqslant \Delta(G, \mathbf{w}) .
$$

The unweighted case corresponds to setting all edge weights to 1 .

Let us make a remark about the treatment of multiple edges. It is easy to see that all the quantities appearing in (2.20) are unchanged if we replace a family $e_{1}, \ldots, e_{n}$ of parallel edges with weights $w_{e_{1}}, \ldots, w_{e_{n}}$ by a single edge $e$ with weight $w_{e}=\sum_{i=1}^{n} w_{e_{i}}$. So, in proving (2.20), we could, if we wanted, restrict attention to simple graphs; but we don't bother, because no simplification of the proof is obtained by doing so. Likewise, the weighted counts discussed in Sections 5 and 6 are unchanged by this replacement, because the subgraphs in question (walks, paths, trees and forests) can include at most one edge from a family of parallel edges. So it would suffice to prove the bounds in Sections 5 and 6 for simple graphs; but once again, we refrain from making this assumption because nothing is gained by doing so. For the weighted counts discussed in Sections 7 and 8, by contrast, no simple reduction of multiple edges can be performed, because the subgraphs in question $d o$ permit the inclusion of multiple edges. We shall therefore have to deal there with multigraphs in all our arguments.

\section{Cocycle Bases and Maxmaxflow}

Given a graph $G$ and disjoint subsets $X, Y \subseteq V(G)$, let $E(X, Y)$ denote the set of edges in $G$ between $X$ and $Y$. A cocycle of $G$ is a set $E(X, Y)$ where $X, Y$ is a bipartition of $V(G)$; note that $X=\varnothing$ and $Y=\varnothing$ are allowed. Let $\oplus$ denote symmetric difference. The following lemma is well known:

Lemma 3.1 Let $C_{1}=E\left(X_{1}, Y_{1}\right)$ and $C_{2}=E\left(X_{2}, Y_{2}\right)$ be two cocycles in $G$. Then $C_{1} \oplus$ $C_{2}=E\left(\left(X_{1} \cap X_{2}\right) \cup\left(Y_{1} \cap Y_{2}\right),\left(X_{1} \cap Y_{2}\right) \cup\left(Y_{1} \cap X_{2}\right)\right)$.

It follows that the set of all cocycles of $G$ forms a vector space over $\operatorname{GF}(2)$ with respect to symmetric difference. This is the cocycle space of $G$. Its dimension is $|V(G)|-c(G)$, where $c(G)$ denotes the number of components of $G$.

Lemma 3.2 Let $G$ be a connected graph and let $C$ be a cocycle of $G$. Then $C$ corresponds to a unique bipartition of $V(G)$.

Proof. Suppose $C=E\left(X_{1}, Y_{1}\right)=E\left(X_{2}, Y_{2}\right)$. Since $C \oplus C=\varnothing$ there are no edges in $G$ from $\left(X_{1} \cap X_{2}\right) \cup\left(Y_{1} \cap Y_{2}\right)$ to $\left(X_{1} \cap Y_{2}\right) \cup\left(Y_{1} \cap X_{2}\right)$. Since $G$ is connected, it follows that either $\left(X_{1} \cap X_{2}\right) \cup\left(Y_{1} \cap Y_{2}\right)=\varnothing$ and hence $\left(X_{1}, Y_{1}\right)=\left(Y_{2}, X_{2}\right)$, or else $\left(X_{1} \cap Y_{2}\right) \cup\left(Y_{1} \cap X_{2}\right)=\varnothing$ and hence $\left(X_{1}, Y_{1}\right)=\left(X_{2}, Y_{2}\right)$. 
Lemma 3.3 Let $G$ be a connected graph, let $C_{1}, C_{2}$ be cocycles of $G$, and let $x, y$ be vertices of $G$. Suppose that $x, y$ belong to the same subset in the bipartitions of $G$ corresponding to $C_{1}$ and $C_{2}$, respectively. Then $x, y$ belong to the same subset in the bipartition of $G$ corresponding to $C_{1} \oplus C_{2}$.

Proof. Immediate from Lemma 3.1.

Lemma 3.4 Let $G$ be a connected graph and let $C_{1}, C_{2}, \ldots, C_{m}$ be cocycles of $G$. Suppose that for each $i$, there exists a pair of vertices $x_{i}, y_{i}$ such that $x_{i}, y_{i}$ belong to different subsets in the bipartition of $G$ corresponding to $C_{i}$ and to the same subset in the bipartition of $G$ corresponding to $C_{j}$ for all $j \neq i(1 \leqslant j \leqslant m)$. Then $C_{1}, C_{2}, \ldots, C_{m}$ are linearly independent.

Proof. If not, then we may suppose without loss of generality that $C_{1}=C_{2} \oplus C_{3} \oplus$ $\ldots \oplus C_{m}$. This contradicts the fact that $x_{1}, y_{1}$ belong to the different subsets in the bipartition corresponding to $C_{1}$ and to the same subset in the bipartition corresponding to $C_{2} \oplus C_{3} \oplus \ldots \oplus C_{m}$, by Lemma 3.3.

Let $G$ be a connected graph and let $T$ be a tree on the same vertex set $V$ as $G$. (We emphasize that $T$ need not be a subgraph of $G$.) Each edge $e \in E(T)$ induces a bipartition of $V$ into nonempty subsets $X, Y$ given by the two components of $T-e$; we define the elementary cocycle of $G$ corresponding to $e$ and $T$ to be the cocycle $E_{G}(X, Y)$.

Lemma 3.5 Let $G$ be a connected graph with $n$ vertices, and let $T$ be a tree on the same set of vertices (not necessarily a subgraph of $G$ ). For each edge $e_{i} \in E(T)$, let $C_{i}$ be the elementary cocycle of $G$ corresponding to $e_{i}$ and $T$. Then $\left\{C_{1}, C_{2}, \ldots, C_{n-1}\right\}$ is a basis for the cocycle space of $G$.

Proof. Using Lemma 3.4 (taking $x_{i}, y_{i}$ to be the end-vertices of $e_{i}$ ) we deduce that $C_{1}, C_{2}, \ldots, C_{n-1}$ are linearly independent. Since the dimension of the cocycle space of $G$ is $n-1$, they form a basis.

Lemma 3.6 Let $G$ be a connected graph with $n$ vertices, let $\left\{C_{1}, C_{2}, \ldots, C_{n-1}\right\}$ be a basis for the cocycle space of $G$, and let $x, y \in V(G)$ with $x \neq y$. Then $x, y$ belong to different subsets in the bipartition corresponding to $C_{i}$, for some $1 \leqslant i \leqslant n-1$.

Proof. Suppose not. Let $C$ be a cocycle in $G$ that separates $x$ and $y$ [for example, $\left.E\left(\{x\},\{x\}^{c}\right)\right]$. Since $\left\{C_{1}, C_{2}, \ldots, C_{n-1}\right\}$ is a basis for the cocycle space of $G, C$ is a linear combination of $C_{1}, C_{2}, \ldots, C_{n-1}$. This contradicts Lemma 3.3.

Now let $G$ be equipped with a family of nonnegative real edge weights $\mathbf{w}=\left\{w_{e}\right\}_{e \in E(G)}$. As in $(2.18) /(2.19)$, we let $\lambda_{G}(x, y ; \mathbf{w})$ be the max flow from $x$ to $y$ with edge capacities $\mathbf{w}$, and $\Lambda(G, \mathbf{w})$ the corresponding maxmaxflow. As in $(2.16)$, we let $\widetilde{\Lambda}(G, \mathbf{w})$ be the minmax weight of the cocycles in a basis. In order to prove the fundamental result (2.20), we shall need the following classic result on flows (see [8, Section 2.3] for an excellent exposition): 
Theorem 3.7 (Gomory and $\mathbf{H u}[4]$ ) Let $G$ be a connected graph equipped with nonnegative real edge weights $\mathbf{w}=\left\{w_{e}\right\}_{e \in E(G)}$. Then there exists a tree $T$ with vertex set $V(T)=V(G) \equiv V$ (note that $T$ is not necessarily a subgraph of $G$ !) and a set $\mathbf{w}^{T}=\left\{w_{e}^{T}\right\}_{e \in E(T)}$ of nonnegative real edge weights such that

(a) $\lambda_{G}(x, y ; \mathbf{w})=\lambda_{T}\left(x, y ; \mathbf{w}^{T}\right)$ for all $x, y \in V(x \neq y)$, and

(b) for each $e=x y \in E(T)$, the elementary cocycle $C$ of $G$ corresponding to $e$ and $T$ is a minimum-weight edge cut separating $x$ from $y$ in $G$, i.e. $\lambda_{G}(x, y ; \mathbf{w})=\sum_{f \in C} w_{f}$.

We shall call any tree $T$ with the above properties a Gomory-Hu tree for $(G, \mathbf{w})$; it is in general nonunique. Note that, for any given tree $T$, there is at most one choice of $\mathbf{w}^{T}$ that satisfies (a), namely for each edge $e=x y \in E(T)$ we must set $w_{e}^{T}=\lambda_{G}(x, y ; \mathbf{w})$. It can also be shown that if $T$ satisfies (b), then this definition of $\mathbf{w}^{T}$ necessarily satisfies (a); but we shall not need this fact.

If $T$ is a Gomory-Hu tree for $(G, \mathbf{w})$, we define $\widehat{\Lambda}(G, \mathbf{w} ; T)=\max _{e \in E(T)} w_{e}^{T}$. We claim that this value is independent of the choice of $T$, and in fact we have:

Theorem 3.8 Let $G$ be a connected graph equipped with nonnegative real edge weights $\mathbf{w}=\left\{w_{e}\right\}_{e \in E(G)}$, and let $T$ be a Gomory-Hu tree for $(G, \mathbf{w})$. Then

$$
\Lambda(G, \mathbf{w})=\widehat{\Lambda}(G, \mathbf{w} ; T)=\widetilde{\Lambda}(G, \mathbf{w}) \leqslant \Delta_{2}(G, \mathbf{w}) \leqslant \Delta(G, \mathbf{w}) .
$$

In particular, the value of $\widehat{\Lambda}(G, \mathbf{w} ; T)$ is independent of the choice of $T$.

Proof. The equality $\Lambda(G, \mathbf{w})=\Lambda\left(T, \mathbf{w}^{T}\right)$ follows from Theorem 3.7(a), and it is trivial to see that $\Lambda\left(T, \mathbf{w}^{T}\right)=\max _{e \in E(T)} w_{e}^{T}$. This proves that $\Lambda(G, \mathbf{w})=\widehat{\Lambda}(G, \mathbf{w} ; T)$ and in particular that the latter quantity is independent of the choice of $T$.

The inequality $\Lambda(G, \mathbf{w}) \leqslant \widetilde{\Lambda}(G, \mathbf{w})$ follows from Lemma 3.6.

The inequality $\widetilde{\Lambda}(G, \mathbf{w}) \leqslant \widehat{\Lambda}(G, \mathbf{w} ; T)$ follows from Lemma 3.5 and Theorem $3.7(\mathrm{a}, \mathrm{b})$.

There are easy elementary proofs of both $\Lambda(G, \mathbf{w}) \leqslant \Delta_{2}(G, \mathbf{w})$ and $\widetilde{\Lambda}(G, \mathbf{w}) \leqslant$ $\Delta_{2}(G, \mathbf{w})$, as noted in the Introduction.

Corollary 3.9 Let $G$ be a (not necessarily connected) graph equipped with nonnegative real edge weights $\mathbf{w}=\left\{w_{e}\right\}_{e \in E(G)}$. Then

$$
\Lambda(G, \mathbf{w})=\widetilde{\Lambda}(G, \mathbf{w}) \leqslant \Delta_{2}(G, \mathbf{w}) \leqslant \Delta(G, \mathbf{w}) .
$$

Proof. If $G$ is disconnected, it suffices to apply Theorem 3.8 to each component of $G$. 
Finally, we need to prove our claims that $\Lambda(G, \mathbf{w}) \geqslant D(G, \mathbf{w})$ and $\Lambda(G, \mathbf{w}) \geqslant$ $\Delta_{n-1}(G, \mathbf{w})$. We shall actually prove a slightly stronger result. Define the $k^{\text {th }}$ weighted degeneracy number

$$
D_{k}(G, \mathbf{w})=\max _{H \subseteq G} \delta_{k}(H, \mathbf{w}),
$$

where the max runs over all subgraphs $H$ of $G$, and $\delta_{k}(H, \mathbf{w})$ denotes the $k$ th smallest weighted degree of $H$ :

$$
\delta_{k}(H, \mathbf{w})=\max _{x_{1}, \ldots, x_{k-1} \in V(H)} \min _{x \in V(H) \backslash\left\{x_{1}, \ldots, x_{k-1}\right\}} d_{H}(x, \mathbf{w}) .
$$

Trivially we have $D(G, \mathbf{w}) \equiv D_{1}(G, \mathbf{w}) \leqslant D_{2}(G, \mathbf{w}) \leqslant \ldots$ and $\delta_{k}(G, \mathbf{w}) \leqslant D_{k}(G, \mathbf{w})$. In particular,

$$
D_{2}(G, \mathbf{w}) \geqslant \max \left[D(G, \mathbf{w}), \delta_{2}(G, \mathbf{w})\right] .
$$

Proposition 3.10 Let $G$ be a graph with $n$ vertices $(n \geqslant 2)$ equipped with nonnegative real edge weights $\mathbf{w}=\left\{w_{e}\right\}_{e \in E(G)}$. Then

$$
\Lambda(G, \mathbf{w}) \geqslant D_{2}(G, \mathbf{w}) \geqslant \max \left[D(G, \mathbf{w}), \Delta_{n-1}(G, \mathbf{w})\right] .
$$

Proof. Suppose first that $G$ is connected, and let $T$ be a Gomory-Hu tree for $(G, \mathbf{w})$. For any vertex $x$ of degree 1 in $T$, let $e=x y$ be the unique incident edge in $T$; then the elementary cocycle of $G$ corresponding to $e$ and $T$ is $E_{G}\left(\{x\},\{x\}^{c}\right)$. Using Theorem 3.7(b), we have $\Lambda(G, \mathbf{w}) \geqslant \lambda_{G}(x, y ; \mathbf{w})=d_{G}(x, \mathbf{w})$. Since there are at least two such vertices $x$, we have $\Lambda(G, \mathbf{w}) \geqslant \delta_{2}(G, \mathbf{w})$.

If $G$ is disconnected, we can apply the result just proven to each component of $G$; we conclude again that $\Lambda(G, \mathbf{w}) \geqslant \delta_{2}(G, \mathbf{w})$.

Now apply this result to each subgraph $H$ of $G$ : we conclude that $\Lambda\left(H,\left.\mathbf{w}\right|_{H}\right) \geqslant$ $\delta_{2}(H, \mathbf{w})$. But $\Lambda(G, \mathbf{w}) \geqslant \Lambda\left(H,\left.\mathbf{w}\right|_{H}\right)$ for every subgraph $H$ of $G$, so $\Lambda(G, \mathbf{w}) \geqslant D_{2}(G, \mathbf{w})$.

Let us now prove a few further general properties of maxmaxflow. Let $G$ be a graph and $x \in V(G)$. We say that $x$ is a cut vertex of $G$ if $G \backslash x$ has more components than $G$. We say that $G$ is non-separable if $G$ is connected and has no cut vertices. ${ }^{5}$ A block of $G$ is a maximal non-separable subgraph of $G$. We first observe that maxmaxflow has a naturalness property that maximum degree and $k$ th-largest degree lack, namely, it "trivializes over blocks":

Proposition 3.11 Let $G_{1}, \ldots, G_{b}$ be the blocks of $G$. Then $\Lambda(G, \mathbf{w})=\max _{1 \leqslant i \leqslant b} \Lambda\left(G_{i}, \mathbf{w}\right)$.

\footnotetext{
${ }^{5}$ This concept is closely related to the more common notion of 2-connectedness. A graph $G$ is 2connected if $G$ has at least three vertices and $G \backslash x$ is connected for all $x \in V(G)$. Thus, a graph with at least three vertices is non-separable if and only if it is 2-connected. However, the graphs $K_{1}$ (a single vertex with no edges) and $K_{2}^{(m)}$ (a pair of vertices connected by $m$ parallel edges, with $m \geqslant 1$ ) are non-separable but not 2-connected.
} 
Proof. If $x$ and $y$ lie in the same block $G_{i}$, then $\lambda_{G}(x, y ; \mathbf{w})=\lambda_{G_{i}}(x, y ; \mathbf{w})$. If $x$ and $y$ lie in the same component of $G$ but in different blocks, then there exist cut vertices $v_{1}, \ldots, v_{k}$ of $G$ and blocks $G_{i_{0}}, G_{i_{1}}, \ldots, G_{i_{k}}$ of $G$ such that $x \in V\left(G_{i_{0}}\right), y \in V\left(G_{i_{k}}\right)$, $V\left(G_{i_{j-1}}\right) \cap V\left(G_{i_{j}}\right)=\left\{v_{j}\right\}$, and every path from $x$ to $y$ passes through $v_{1}, \ldots, v_{k}$ in that order; and in this case we have

$$
\lambda_{G}(x, y ; \mathbf{w})=\min \left[\lambda_{G_{0}}\left(x, v_{1} ; \mathbf{w}\right), \lambda_{G_{1}}\left(v_{1}, v_{2} ; \mathbf{w}\right), \ldots, \lambda_{G_{k-1}}\left(v_{k-1}, v_{k} ; \mathbf{w}\right), \lambda_{G_{k}}\left(v_{k}, y ; \mathbf{w}\right)\right] .
$$

Finally, if $x$ and $y$ lie in different components of $G$, then $\lambda_{G}(x, y ; \mathbf{w})=0$.

It follows immediately from the definition of maxmaxflow that for any pair of distinct vertices $x_{1}, x_{2}$ of $G$, there exists a partition $V(G)=X_{1} \cup X_{2}$ such that $x_{1} \in X_{1}, x_{2} \in X_{2}$ and $\sum_{e \in E\left(X_{1}, X_{2}\right)} w_{e}=\lambda_{G}\left(x_{1}, x_{2} ; \mathbf{w}\right) \leqslant \Lambda(G, \mathbf{w})$. We will need the following extension of this observation in Section 8.

Proposition 3.12 Let $X \subseteq V(G)$ with $|X| \geqslant 2$. Then there exist $x_{1}, x_{2} \in X$ and disjoint $X_{1}, X_{2} \subseteq V(G)$ such that $X \cap X_{i}=\left\{x_{i}\right\}$ and $\sum_{e \in E\left(X_{i}, X_{i}^{c}\right)} w_{e} \leqslant \Lambda(G, \mathbf{w})$ for all $1 \leqslant i \leqslant 2$.

Proof. We can assume without loss of generality that $G$ is connected. Let $T$ be a Gomory-Hu tree for $(G, \mathbf{w})$, and let $T^{\prime}$ be the union of all the paths in $T$ connecting pairs of vertices of $X$. Let $x_{1}, x_{2}$ be distinct end-vertices of $T^{\prime}$ and $e_{1}, e_{2}$ be the edges of $T^{\prime}$ incident with $x_{1}, x_{2}$ respectively. For $i=1,2$, let $X_{i}$ be the vertex set of the component of $T \backslash e_{i}$ which contains $x_{i}$. Then $X_{1} \cap X_{2}=\varnothing$. Furthermore, $E\left(X_{i}, X_{i}^{c}\right)$ is the elementary cocycle of $G$ corresponding to the edge $e_{i}$ of $T$; so by Theorem 3.7(b) we have $\sum_{e \in E\left(X_{i}, X_{i}^{c}\right)} w_{e} \leqslant \Lambda(G, \mathbf{w})$ for $i=1,2$.

We conclude this section with a few examples of maxmaxflow calculations:

Example 3.1. Let $G$ be any forest, and let $\mathbf{w}$ be any set of nonnegative edge weights. Then $\Lambda(G, \mathbf{w})=\max _{e \in E(G)} w_{e}$. Indeed, this elementary fact was already used in the proof of Theorem 3.8.

Example 3.2. Let $G$ be the cycle $C_{n}$, and let $\mathbf{w}$ be any set of nonnegative edge weights. Then

$$
\Lambda(G, \mathbf{w})=\max _{e \in E(G)} w_{e}+\min _{e \in E(G)} w_{e} .
$$

Proof. For any pair of distinct vertices $x, y \in V(G)$, there are precisely two paths from $x$ to $y$, and together they use all the edges of $G$. Hence on one path the max flow is exactly $\min w_{e}$, and on the other it is at most $\max w_{e}$. So $\Lambda(G, \mathbf{w}) \leqslant \min w_{e}+\max w_{e}$. On the other hand, if we take $x, y$ to be the endpoints of the edge with maximum weight, we obtain equality. 


\section{Some Preliminaries}

Before turning to the counting of subgraphs of various classes, let us make some brief prefatory observations.

\subsection{Pointwise bounds vs. generating-function bounds}

Let $(G, \mathbf{w})$ be a weighted graph, and suppose that $a_{m}$ is the total weight of all $m$-edge subgraphs of $G$ of some specified class. In the following sections we shall prove upper bounds on $a_{m}$ of two different types:

- "Pointwise bound": $a_{m} \leqslant C_{m}$ for some specified constants $C_{m}$.

- "Generating-function bound": $\sum_{m=0}^{\infty} a_{m} z^{m} \leqslant C(z)$ for some specified function $C(z)$ [which is allowed to take the value $+\infty$ ], for all $z \geqslant 0$.

From each type of bound we can deduce one of the other type: $a_{m} \leqslant C_{m}$ trivially implies $\sum a_{m} z^{m} \leqslant \sum C_{m} z^{m} ;$ and $\sum a_{m} z^{m} \leqslant C(z)$ implies $a_{m} \leqslant \inf _{z \geqslant 0} z^{-m} C(z)$. But we lose something in each such passage: for example,

$$
\sum_{m=0}^{\infty} a_{m} \leqslant 1 \Longrightarrow a_{m} \leqslant 1 \Longrightarrow \sum_{m=0}^{\infty} a_{m} z^{m} \leqslant \frac{1}{1-z} \Longrightarrow a_{m} \leqslant \frac{(m+1)^{m+1}}{m^{m}} \Longrightarrow \ldots
$$

So it is important to choose the best type of bound in each problem. Roughly speaking, the pointwise bound is better in those cases where the bound can be saturated simultaneously for all (or arbitrarily many) values of $m$. By contrast, in cases where the pointwise bound can be saturated only for one value of $m$ at a time, it may be possible to prove a stronger bound of generating-function type. These remarks will be illustrated repeatedly throughout the following sections.

\subsection{Convex hulls in graphs}

Let $H$ be any graph, and let $X$ be a nonempty subset of $V(H)$. We define the convex hull of $X$ in $H$, denoted $\operatorname{conv}(X, H)$, to be the union of all paths in $H$ connecting any pair of vertices $x_{1}, x_{2} \in X$ (including paths of length 0 from a vertex $x \in X$ to itself). Thus, $\operatorname{conv}(X, H)$ is a subgraph of $H$ whose vertex set contains $X$. The following properties are elementary consequences of this definition:

(Conv1) If $H$ is connected, then $\operatorname{conv}(X, H)$ is connected.

(Conv2) Any vertex of degree 0 or 1 in $\operatorname{conv}(X, H)$ must belong to $X$. Moreover, a vertex $x \in X$ is of degree 0 (i.e. isolated) in $\operatorname{conv}(X, H)$ if and only if it is the only element of $X$ in its component of $H$.

(Conv3) If $H$ is a tree, then $\operatorname{conv}(X, H)$ is the smallest subtree of $H$ containing all the vertices of $X$. 
(Conv4) If $H$ is non-separable and $|X| \geqslant 2$, then $\operatorname{conv}(X, H)=H$. [This is because, for any pair of distinct vertices $x_{1}, x_{2}$ of $H$, every edge of $H$ lies on some path from $x_{1}$ to $x_{2}$.]

Property (Conv4) can be generalized as follows. Let $H$ be a graph and $x \in V(H)$. We say that $x$ is an internal vertex of $H$ if $x$ is not a cut vertex of $H$. An end block of $H$ is a block that contains exactly one cut vertex of $H$.

Lemma 4.1 Let $H$ be connected, and let $X \subseteq V(H)$ with $|X| \geqslant 2$. Then:

(a) $\operatorname{conv}(X, H)$ is a connected union of blocks of $H$.

(b) Each end block of $\operatorname{conv}(X, H)$ has an internal vertex belonging to $X$.

Proof. This follows easily from the definition of $\operatorname{conv}(X, H)$ and property (Conv4).

We have already used convex hulls in the proof of Proposition 3.12, and they will play an important role in our treatment of trees and block trees (Sections 6 and 8).

\section{Counting Walks and Paths}

Let $G$ be a graph equipped with nonnegative real edge weights $\mathbf{w}=\left\{w_{e}\right\}_{e \in E(G)}$. In this section (as well as in the following ones) we shall write $\Delta, \Delta_{2}, \Lambda, \ldots$ as a shorthand for $\Delta(G, \mathbf{w}), \Delta_{2}(G, \mathbf{w}), \Lambda(G, \mathbf{w}), \ldots$; the underlying graph $G$ and its edge weights $\mathbf{w}$ will always be understood. Similarly, we shall write $\lambda(x, y)$ as a shorthand for $\lambda_{G}(x, y ; \mathbf{w})$.

For $x, y \in V(G)$ and $m \geqslant 0$, let $\mathcal{W}_{m}(x, y)$ be the set of $m$-step walks from $x$ to $y$, i.e. sequences $\omega=x_{0} e_{1} x_{1} e_{2} x_{2} \cdots x_{m-1} e_{m} x_{m}$ with $x_{0}=x$ and $x_{m}=y$ such that each $e_{i}$ is an edge $x_{i-1} x_{i}$. We then define the following subsets of $\mathcal{W}_{m}(x, y)$ :

- $\mathcal{W}_{m}^{\mathrm{FPW}}(x, y)$ consists of first-passage walks, i.e. walks such that $x_{i} \neq y$ for $i<m$;

- $\mathcal{W}_{m}^{\mathrm{SAW}}(x, y)$ consists of self-avoiding walks (otherwise known as paths), i.e. walks such that $x_{i} \neq x_{j}$ for $i \neq j$. [We remark that a walk $\omega$ is self-avoiding if and only if each initial segment of $\omega$ is a first-passage walk.]

Clearly $\mathcal{W}_{m}^{\mathrm{SAW}}(x, y) \subseteq \mathcal{W}_{m}^{\mathrm{FPW}}(x, y) \subseteq \mathcal{W}_{m}(x, y)$.

More generally, let $x \in V(G)$ and $Y \subseteq V(G)$. We let $\mathcal{W}_{m}(x, Y)=\bigcup_{y \in Y} \mathcal{W}_{m}(x, y)$ be the set of $m$-step walks from $x$ to $Y$. We then define the following subsets of $\mathcal{W}_{m}(x, Y)$ :

- $\mathcal{W}_{m}^{\mathrm{FPW}}(x, Y)$ consists of first-passage walks to the set $Y$, i.e. walks such that $x_{i} \notin Y$ for $i<m$;

- $\mathcal{W}_{m}^{\mathrm{FPSAW}}(x, Y)$ consists of first-passage self-avoiding walks to the set $Y$, i.e. walks such that $x_{i} \notin Y$ for $i<m$ and $x_{i} \neq x_{j}$ for $i \neq j$. 
Then $\mathcal{W}_{m}^{\text {FPSAW }}(x, Y) \subseteq \mathcal{W}_{m}^{\mathrm{FPW}}(x, Y) \subseteq \mathcal{W}_{m}(x, Y)$, and in fact $\mathcal{W}_{m}^{\text {FPSAW }}(x, Y)=$ $\mathcal{W}_{m}^{\mathrm{FPW}}(x, Y) \cap \bigcup_{y \in Y} \mathcal{W}_{m}^{\mathrm{SAW}}(x, y)$.

We define the weight of a walk $\omega=x_{0} e_{1} x_{1} e_{2} x_{2} \cdots x_{m-1} e_{m} x_{m}$ to be the product of its edge weights:

$$
w\left(x_{0} e_{1} x_{1} e_{2} x_{2} \cdots x_{m-1} e_{m} x_{m}\right)=\prod_{i=1}^{m} w_{e_{i}} .
$$

Finally, we define the weighted counts

$$
\begin{aligned}
w_{m}(x, y) & =\sum_{\omega \in \mathcal{W}_{m}(x, y)} w(\omega) \\
w_{m}(x, Y) & =\sum_{\omega \in \mathcal{W}_{m}(x, Y)} w(\omega)
\end{aligned}
$$

and likewise for $w^{\mathrm{FPW}}, w^{\mathrm{SAW}}$ and $w^{\mathrm{FPSAW}}$.

The weighted count of walks with a fixed initial vertex and an arbitrary final vertex can trivially be bounded in terms of maximum weighted degree:

Proposition 5.1 For all $x \in V(G)$ and all $m \geqslant 0$, we have

$$
\sum_{y \in V(G)} w_{m}(x, y) \leqslant \Delta^{m}
$$

In fact, when $(G, \mathbf{w})$ is $\Delta$-regular [i.e. $d_{G}(x, \mathbf{w})=\Delta$ for all $x \in V(G)$ ], the bound (5.4) is sharp simultaneously for all $x \in V(G)$ and all $m \geqslant 0$.

If we restrict to first-passage walks to a fixed target set $Y$, we can obtain a "generatingfunction" bound that is sharper than (5.4):

Proposition 5.2 For all $x \in V(G)$ and all $Y \subseteq V(G)$, we have

$$
\sum_{m=0}^{\infty} \Delta^{-m} w_{m}^{\mathrm{FPW}}(x, Y) \leqslant 1 .
$$

Proof. Define a sub-Markov chain ${ }^{6}$ with transition probabilities $p\left(x \rightarrow x^{\prime}\right)=\Delta^{-1} \sum_{e=x x^{\prime}} w_{e}$ (here the sum runs over all edges $e=x x^{\prime}$ in $G$ ). Then $\sum_{m=0}^{\infty} \Delta^{-m} w_{m}^{\mathrm{FPW}}(x, Y)$ is the probability that $Y$ is eventually hit, starting at $x$.

Remarks. 1. If $G$ is connected (with weights $\left.w_{e}>0\right)$ and $(G, \mathbf{w})$ is $\Delta$-regular, then (5.5) is equality for all $x \in V(G)$ and all nonempty $Y \subseteq V(G)$. This is because $p$ is a finite

\footnotetext{
${ }^{6} \mathrm{~A}$ sub-Markov chain on a finite or countably infinite state space $X$ is defined by a transition kernel $\left\{p\left(x \rightarrow x^{\prime}\right)\right\}_{x, x^{\prime} \in X}$ satisfying $p\left(x \rightarrow x^{\prime}\right) \geqslant 0$ and $\sum_{x^{\prime} \in X} p\left(x \rightarrow x^{\prime}\right) \leqslant 1$. It induces a Markov chain on the state space $X \cup\{\infty\}$ (where the new state $\infty \notin X$ is called the "cemetery") by defining $p(x \rightarrow \infty)=$ $1-\sum_{x^{\prime} \in X} p\left(x \rightarrow x^{\prime}\right), p(\infty \rightarrow x)=0$ for all $x \in X$, and $p(\infty \rightarrow \infty)=1$.
} 
irreducible Markov chain, so that $Y$ is hit with probability 1. Indeed, it suffices to have $d_{G}(x, \mathbf{w})=\Delta$ for all $x \in V(G) \backslash Y$; the degree at vertices of $Y$ is irrelevant.

2. If $x \in Y$ then $w_{0}^{\mathrm{FPW}}(x, Y)=1$ and $w_{m}^{\mathrm{FPW}}(x, Y)=0$ for $m \geqslant 1$ so equality holds in (5.5). On the other hand, if $x \notin Y$, then we can improve the upper bound in (5.5) from 1 to $d_{G}(x, \mathbf{w}) / \Delta$, since this is the probability for the Markov chain to survive the first step.

3. As just mentioned, (5.5) is the best-possible upper bound for first-passage walks to a set $Y$. However, one might ask whether a sharper bound is possible by restricting attention to first-passage self-avoiding walks. The answer is no, at least if one considers a general set $Y$, as the following examples show:

Example 5.1. Let $G$ be the star $K_{1, r}$, let $x$ be the central vertex, and let $Y$ be the remaining vertices. Set all edge weights $w_{e}=\Delta / r$. Then $w_{1}^{\mathrm{FPSAW}}(x, Y)=\Delta$ and $w_{m}^{\mathrm{FPSAW}}(x, Y)=0$ for $m \neq 1$, so (5.5) is sharp.

Example 5.2. More generally, let $\mathbf{T}_{r}$ be the infinite $r$-regular tree, let $x$ be any vertex of $\mathbf{T}_{r}$, and fix $n \geqslant 1$. Let $G$ be the subtree of $\mathbf{T}_{r}$ induced by the vertices lying at a distance at most $n$ from $x$, and let $Y$ be the set of vertices lying at a distance exactly $n$ from $x$. Set all edge weights $w_{e}=\Delta / r$. Then

$$
w_{m}^{\mathrm{FPSAW}}(x, Y)= \begin{cases}r(r-1)^{m-1}(\Delta / r)^{m} & \text { if } m=n \\ 0 & \text { otherwise }\end{cases}
$$

so that

$$
\sum_{m=0}^{\infty} \Delta^{-m} w_{m}^{\mathrm{FPSAW}}(x, Y)=\left(\frac{r-1}{r}\right)^{n-1},
$$

which is sharp in the limit $r \rightarrow \infty$ at any fixed $n$. (It is also sharp for any $r$ when $n=1$, which is the case $G=K_{1, r}$.)

For comparison with Proposition 5.3 below, it is perhaps illuminating to rephrase the proof of Proposition 5.2 as an induction:

SECOND PROOF OF Proposition 5.2. We will prove inductively that

$$
\sum_{m=0}^{M} \Delta^{-m} w_{m}^{\mathrm{FPW}}(x, Y) \leqslant 1
$$

for all $M \geqslant 0$ and all $x \in V(G)$. This clearly holds for $M=0$. Moreover, it holds for all $M$ when $x \in Y$, since $w_{m}^{\mathrm{FPW}}(x, Y)=\delta_{m 0}$. So suppose that $x \notin Y$. Then $w_{0}^{\mathrm{FPW}}(x, Y)=0$ and for $m \geqslant 1$ we have

$$
w_{m}^{\mathrm{FPW}}(x, Y)=\sum_{x^{\prime} \in V(G)}\left(\sum_{e=x x^{\prime}} w_{e}\right) w_{m-1}^{\mathrm{FPW}}\left(x^{\prime}, Y\right) .
$$


Thus, for $M \geqslant 1$, we have

$$
\begin{aligned}
\sum_{m=0}^{M} \Delta^{-m} w_{m}^{\mathrm{FPW}}(x, Y) & =\sum_{m=1}^{M} \Delta^{-m} \sum_{x^{\prime} \in V(G)}\left(\sum_{e=x x^{\prime}} w_{e}\right) w_{m-1}^{\mathrm{FPW}}\left(x^{\prime}, Y\right) \\
& =\Delta^{-1} \sum_{x^{\prime} \in V(G)}\left(\sum_{e=x x^{\prime}} w_{e}\right) \sum_{j=0}^{M-1} \Delta^{-j} w_{j}^{\mathrm{FPW}}\left(x^{\prime}, Y\right) \\
& \leqslant 1 \text { by the inductive hypothesis. }
\end{aligned}
$$

There is no hope of obtaining a $|Y|$-independent bound on $w_{m}^{\mathrm{FPW}}(x, Y)$ - or even on $w_{m}^{\text {FPSAW }}(x, Y)$ - in terms of second-largest degree (much less in terms of maxmaxflow). Indeed, let $G$ be the star $K_{1, r}$, so that $\Delta=r$ and $\Delta_{2}=\Lambda=1$; let $x$ be the central vertex and $Y$ the remaining vertices; then $w_{1}^{\text {FPSAW }}(x, Y)=r$, which is unbounded. Nor can we bound $w_{m}^{\mathrm{FPW}}(x, y)$ : considering again the star and taking $y$ to be any vertex other than $x$, we have $w_{3}^{\mathrm{FPW}}(x, y)=r-1$, which is again unbounded. Nevertheless, an analogue of Proposition 5.2 does hold for maxmaxflow if we restrict ourselves to self-avoiding walks and to the case $Y=\{y\}$ :

Proposition 5.3 Define

$$
F(x, y)= \begin{cases}\lambda(x, y) / \Lambda & \text { if } x \neq y \\ 1 & \text { if } x=y\end{cases}
$$

Then, for all $x, y \in V(G)$, we have

$$
\sum_{m=0}^{\infty} \Lambda^{-m} w_{m}^{\mathrm{SAW}}(x, y) \leqslant F(x, y) \leqslant 1 .
$$

Proof. We will prove inductively that $\sum_{m=0}^{M} \Lambda^{-m} w_{m}^{\mathrm{SAW}}(x, y) \leqslant F(x, y)$ for all $M \geqslant 0$ and all $x, y \in V(G)$. This clearly holds for $M=0$, since $w_{0}^{\mathrm{SAW}}(x, y)=\delta_{x y}$. Moreover, it holds for all $M$ when $x=y$, since $w_{m}^{\mathrm{SAW}}(x, x)=\delta_{m 0}$. So let $M \geqslant 1$ and $x \neq y$. Let $C=E(X, Y)$ be a cocycle in $G$ with $x \in X, y \in Y$ and $\sum_{e \in C} w_{e}=\lambda(x, y)$. Let $e=u v$ be an edge in $C$ with $u \in X$ and $v \in Y$, and let $w_{m}^{\mathrm{SAW}}(x, y, e)$ be the weighted sum over $m$-step paths from $x$ to $y$ that use $e$ as their first edge from $X$ to $Y$. Then

$$
w_{m}^{\mathrm{SAW}}(x, y, e) \leqslant w_{e} \sum_{i=0}^{m-1} w_{i}^{\mathrm{SAW}}(x, u) w_{m-1-i}^{\mathrm{SAW}}(v, y) .
$$

So

$$
\sum_{m=0}^{M} \Lambda^{-m} w_{m}^{\mathrm{SAW}}(x, y, e) \leqslant \Lambda^{-1} w_{e} \sum_{m=0}^{M} \sum_{i=0}^{m-1} \Lambda^{-i} w_{i}^{\mathrm{SAW}}(x, u) \Lambda^{-(m-1-i)} w_{m-1-i}^{\mathrm{SAW}}(v, y)
$$




$$
\begin{aligned}
& =\Lambda^{-1} w_{e} \sum_{i=0}^{M-1} \Lambda^{-i} w_{i}^{\mathrm{SAW}}(x, u) \sum_{j=0}^{M-1-i} \Lambda^{-j} w_{j}^{\mathrm{SAW}}(v, y) \\
& \leqslant \Lambda^{-1} w_{e} \text { by the inductive hypothesis }
\end{aligned}
$$

(note that we may have here used the $x=y$ case of the inductive hypothesis, in case $x=u$ or $y=v$ or both). Therefore

$$
\begin{aligned}
\sum_{m=0}^{M} \Lambda^{-m} w_{m}^{\mathrm{SAW}}(x, y) & =\sum_{e \in C} \sum_{m=0}^{M} \Lambda^{-m} w_{m}^{\mathrm{SAW}}(x, y, e) \\
& \leqslant \sum_{e \in C} \Lambda^{-1} w_{e} \\
& \leqslant \lambda(x, y) / \Lambda .
\end{aligned}
$$

Corollary $5.4 w_{m}^{\mathrm{SAW}}(x, y) \leqslant \Lambda^{m} F(x, y)$ for all $m \geqslant 0$ and all $x, y \in V(G)$.

Corollary 5.5 For $0 \leqslant \zeta \leqslant \Lambda^{-1}$, we have $\sum_{m=0}^{\infty} \zeta^{m} w_{m}^{\mathrm{SAW}}(x, y) \leqslant(\zeta \Lambda)^{\operatorname{dist}(x, y)} F(x, y)$ where $\operatorname{dist}(x, y)$ is the length of the shortest path in $G$ from $x$ to $y$. (Of course, if there is no such path, then $w_{m}^{\mathrm{SAW}}(x, y)=0$ for all $m$.)

Example 5.3. Let $G$ be any tree, let $w_{e}=w$ for all edges $e$, and let $x, y$ be any two vertices of $G$. Then $\lambda(x, y)=\Lambda=w$ and

$$
w_{m}^{\mathrm{SAW}}(x, y)= \begin{cases}w^{m} & \text { if } m=\operatorname{dist}(x, y) \\ 0 & \text { otherwise }\end{cases}
$$

so that (5.11) is equality for all $x, y$. Note that the generating-function bound (5.11) is here much sharper than the pointwise bound of Corollary 5.4: this reflects the fact that the latter can be saturated here for only one value of $m$ (for any given pair $x, y$ ). This is the prototypical situation in which we shall seek generating-function bounds. Indeed, the inductive "cutting" argument used in the proof of Proposition 5.3 seems to work only for the generating-function bound; we do not know of any way of proving Corollary 5.4 without proving the stronger bound (5.11).

Example 5.4. Let $G$ be the complete graph $K_{n}$, and let all edge weights $w_{e}$ equal $\Delta /(n-1)$, so that both the maximum weighted degree and the maxmaxflow equal $\Delta$. If $x, y$ are any two distinct vertices, we have

$$
w_{m}^{\mathrm{SAW}}(x, y)=\frac{(n-2)(n-3) \cdots(n-m)}{(n-1)^{m}} \Delta^{m}
$$


if $1 \leqslant m \leqslant n-1$, and 0 otherwise. Approximating Riemann sums by integrals, we find

$$
\sum_{m=0}^{\infty} \Delta^{-m} w_{m}^{\mathrm{SAW}}(x, y) \approx \sqrt{\frac{\pi}{2 n}}
$$

as $n \rightarrow \infty$, so Proposition 5.3 is far from sharp in this limit. Nevertheless, we see that the exponential growth rate $w_{m}^{\mathrm{SAW}}(x, y) \sim \Delta^{m}$ in Proposition 5.3 and Corollary 5.4 cannot be improved. Indeed, if for each fixed $m$ we choose $n$ so as to maximize $w_{m}^{\mathrm{SAW}}(x, y) / \Delta^{m}$, we find that the maximum is achieved at $n \approx m^{2} / 2$ and that the maximum value is $\approx e /\left(2 m^{2}\right)$.

Example 5.5. Let $G$ be the generalized theta graph $\Theta_{1,2, \ldots, r}(r \geqslant 2)$, consisting of a pair of endvertices $a, b$ joined by $r$ internally disjoint paths of lengths $1,2, \ldots, r$. On each path let one edge have weight $w$ and the other edges have weight 1 . Then the maxmaxflow is

$$
\Lambda= \begin{cases}1+w & \text { if } 0 \leqslant w \leqslant 1 /(r-1) \\ r w & \text { if } 1 /(r-1) \leqslant w \leqslant 1 \\ r-1+w & \text { if } w \geqslant 1\end{cases}
$$

while

$$
w_{m}^{\mathrm{SAW}}(a, b)= \begin{cases}w & \text { if } 1 \leqslant m \leqslant r \\ 0 & \text { otherwise }\end{cases}
$$

In particular, at $w=1 /(r-1)$ we have

$$
\sum_{m=0}^{\infty} \Lambda^{-m} w_{m}^{\mathrm{SAW}}(a, b)=1-\left(1-\frac{1}{r}\right)^{r},
$$

which decreases to $1-1 / e \approx 0.632121$ as $r \rightarrow \infty$. So, although the bound of Proposition 5.3 is not sharp in this case, it does at least come within a constant factor of being

sharp in a situation where the maximum contribution from a single value of $m$ goes to zero (the opposite extreme from Examples 5.1 and 5.2).

\section{Counting Trees and Forests}

Let us now extend Propositions 5.2 and 5.3 from paths to trees and forests. In Section 6.1 we consider classes $\mathcal{T}_{m}(X)$ of trees and $\mathcal{F}_{m}(X, Y)$ of forests. In Section 6.2 we consider a larger class $\mathcal{H}_{m}(X)$ of forests.

\subsection{The classes $\mathcal{T}_{m}(X)$ and $\mathcal{F}_{m}(X, Y)$}

For $F$ a forest in $G$, let $L(F)$ denote the set of vertices of degree 0 or 1 in $F$ (also called leaves or end-vertices of $F$ ).

For any nonempty $X \subseteq V(G)$, let $\mathcal{T}_{m}(X)$ be the set of all $m$-edge trees $T$ in $G$ such that $L(T) \subseteq X \subseteq V(T)$. Heuristically, $\mathcal{T}_{m}(X)$ consists of trees whose leaves are "tied down" on the set $X$. Note the following special cases: 
- If $X=\{x\}$, then $\mathcal{T}_{0}(\{x\})$ has as its single element the edgeless graph with vertex set $\{x\}$, and $\mathcal{T}_{m}(\{x\})=\varnothing$ for $m \geqslant 1$.

- $\mathcal{T}_{m}(\{x, y\}) \simeq \mathcal{W}_{m}^{\mathrm{SAW}}(x, y)$ under the natural identification of paths with their induced subgraphs; this holds both for $x \neq y$ and for $x=y$.

- $\mathcal{T}_{0}(X)=\varnothing$ for $|X| \geqslant 2$.

For any $X, Y \subseteq V(G)$ with $Y \neq \varnothing$, let $\mathcal{F}_{m}(X, Y)$ be the set of all $m$-edge forests $F$ in $G$ such that

(F1) $L(F) \subseteq X \cup Y \subseteq V(F)$, and

(F2) each component of $F$ contains exactly one vertex of $Y$.

[Note that $\mathcal{F}_{m}(X, Y)=\mathcal{F}_{m}(X \backslash Y, Y)$, so we can assume without loss of generality, if desired, that $X \cap Y=\varnothing$.] Heuristically, $\mathcal{F}_{m}(X, Y)$ consists of forests whose leaves are "tied down" on the set $X \cup Y$ and whose components are "tied down" on single elements of the set $Y$. We have the following special cases:

- If $X=\varnothing$ (or more generally if $X \subseteq Y$ ), then $\mathcal{F}_{0}(X, Y)$ has as its single element the edgeless graph with vertex set $Y$, and $\mathcal{F}_{m}(X, Y)=\varnothing$ for $m \geqslant 1$.

- If $X=\{x\}$, then each $F \in \mathcal{F}_{m}(\{x\}, Y)$ is the disjoint union of a path $P \in$ $\mathcal{W}_{m}^{\text {FPSAW }}(x, Y)$ [under the natural identification of paths with their induced subgraphs] and the collection $Y \backslash V(P)$ of isolated vertices; this holds both for $x \notin Y$ and for $x \in Y$. We express this isomorphism loosely by writing $\mathcal{F}_{m}(\{x\}, Y) \simeq$ $\mathcal{W}_{m}^{\text {FPSAW }}(x, Y)$.

- For $Y=\{y\}$, we have $\mathcal{F}_{m}(X,\{y\})=\mathcal{T}_{m}(X \cup\{y\})$.

For $H$ a subgraph of $G$, set $w(H)=\prod_{e \in E(H)} w_{e}$. [Note that if $E(H)=\varnothing$, then $w(H)=1$.] Define the weighted counts

$$
\begin{aligned}
t_{m}(X) & =\sum_{T \in \mathcal{T}_{m}(X)} w(T) \\
f_{m}(X, Y) & =\sum_{F \in \mathcal{F}_{m}(X, Y)} w(F)
\end{aligned}
$$

We will obtain two "generating-function" bounds on $f_{m}(X, Y)$ : a sharp bound in terms of the maximum weighted degree $\Delta$, and a slightly weaker bound in terms of the maxmaxflow $\Lambda$.

Proposition 6.1 For all $X, Y \subseteq V(G)$ with $Y \neq \varnothing$, we have

$$
\sum_{m=0}^{\infty} \Delta^{-m} f_{m}(X, Y) \leqslant 1 .
$$


Proposition 6.2 For all $X, Y \subseteq V(G)$ with $Y \neq \varnothing$, we have

$$
\sum_{m=0}^{\infty}(m+|Y|)^{-(|X|-1)} \Lambda^{-m} f_{m}(X, Y) \leqslant|Y| .
$$

Using the identity $\mathcal{F}_{m}(X,\{y\})=\mathcal{T}_{m}(X \cup\{y\})$, we immediately obtain the following corollaries for trees:

Corollary 6.3 For all nonempty $X \subseteq V(G)$, we have

$$
\sum_{m=0}^{\infty} \Delta^{-m} t_{m}(X) \leqslant 1 .
$$

Corollary 6.4 For all nonempty $X \subseteq V(G)$, we have

$$
\sum_{m=0}^{\infty}(m+1)^{-(|X|-2)} \Lambda^{-m} t_{m}(X) \leqslant 1 .
$$

One can envisage two different approaches to proving these bounds for forests and trees. One is to imitate the "cutting" argument employed for walks in the proofs of Propositions 5.2 and 5.3. The other is to exploit the result obtained for walks in Propositions 5.2 and 5.3 , by reducing forests inductively to paths. The latter technique seems to be more appropriate.

In the proof of Proposition 6.1 we shall make use of the "point-to-set" bound of Proposition 5.2. We shall also use the following elementary lemma which splits a forest with $k$ end-vertices into a forest with $k-1$ end-vertices and a path:

Lemma 6.5 Let $G$ be a graph, let $X, Y \subseteq V(G)$ with $Y \neq \varnothing$, let $x \in X \backslash Y$, and let $F \in \mathcal{F}_{m}(X, Y)$. Let $F_{1}$ be the convex hull of $(X \backslash x) \cup Y$ in $F$, and let $P$ be the unique path in $F$ from $x$ to $V\left(F_{1}\right)$. Then $F$ is the edge-disjoint union of $F_{1}$ and $P$; and for some $i(0 \leqslant i \leqslant m)$ we have $F_{1} \in \mathcal{F}_{i}(X \backslash x, Y)$ and $P \in \mathcal{W}_{m-i}^{\mathrm{FPSAW}}\left(x, V\left(F_{1}\right)\right)$. Moreover, the map $F \mapsto\left(F_{1}, P\right)$ is a bijection from $\mathcal{F}_{m}(X, Y)$ onto $\bigcup_{i=0}^{m} \mathcal{F}_{i}(X \backslash x, Y) \times \mathcal{W}_{m-i}^{\text {FPSAW }}\left(x, V\left(F_{1}\right)\right)$.

The proof is a straightforward exercise: let us simply observe that because $x \notin Y$, the component of $F$ containing $x$ must contain a vertex in $Y$, which guarantees that the path $P$ exists; and $P$ is unique because $F$ has no cycles.

Proof of Proposition 6.1. As noted above, we can assume without loss of generality that $X \cap Y=\varnothing$. We use induction on $k=|X|$. For $k=0$ the result is trivial. For $k=1$ the result follows immediately from Proposition 5.2, since $\mathcal{F}_{m}(\{x\}, Y) \simeq \mathcal{W}_{m}^{\text {FPSAW }}(x, Y) \subseteq$ $\mathcal{W}_{m}^{\text {FPW }}(x, Y)$. So suppose $k \geqslant 2$. Let $x$ be any vertex in $X$; by assumption $x \notin Y$. By Lemma 6.5 , given any $F \in \mathcal{F}_{m}(X, Y)$, we can decompose $F$ into a forest $F_{1} \in \mathcal{F}_{i}(X \backslash x, Y)$ 
and a path $P \in \mathcal{W}_{m-i}^{\text {FPSAW }}\left(x, V\left(F_{1}\right)\right)$; and each such pair $\left(F_{1}, P\right)$ arises from a unique $F$ (namely, $F_{1} \cup P$ ). Since $w(F)=w\left(F_{1}\right) w(P)$, we have

$$
\begin{aligned}
\sum_{m=0}^{\infty} & \Delta^{-m} \sum_{F \in \mathcal{F}_{m}(X, Y)} w(F) \\
& =\sum_{m=0}^{\infty} \sum_{i=0}^{m} \Delta^{-i} \sum_{F_{1} \in \mathcal{F}_{i}(X \backslash x, Y)} w\left(F_{1}\right) \Delta^{-(m-i)} \sum_{P \in \mathcal{W}_{m-i}^{\mathrm{FPSAW}}\left(x, V\left(F_{1}\right)\right)} w(P) \\
& =\sum_{i=0}^{\infty} \Delta^{-i} \sum_{F_{1} \in \mathcal{F}_{i}(X \backslash x, Y)} w\left(F_{1}\right) \sum_{j=0}^{\infty} \Delta^{-j} \sum_{P \in \mathcal{W}_{j}^{\mathrm{FPSAW}}\left(x, V\left(F_{1}\right)\right)} w(P) .
\end{aligned}
$$

Now, for each fixed $F_{1}$ we have $\sum_{j=0}^{\infty} \Delta^{-j} \sum_{P \in \mathcal{W}_{j}^{\mathrm{FPSAW}}\left(x, V\left(F_{1}\right)\right)} w(P) \leqslant 1$ by Proposition 5.2, so that

$$
\sum_{m=0}^{\infty} \Delta^{-m} \sum_{F \in \mathcal{F}_{m}(X, Y)} w(F) \leqslant \sum_{i=0}^{\infty} \Delta^{-i} \sum_{F_{1} \in \mathcal{F}_{i}(X \backslash x, Y)} w\left(F_{1}\right) \leqslant 1
$$

by the inductive hypothesis.

Examples 5.1 and 5.2 show that Proposition 6.1 is best possible (at least for a general set $Y$ ), even when $|X|=1$.

Proof of Proposition 6.2. As before, we assume that $X \cap Y=\varnothing$ and we use induction on $k=|X|$. If $k=0$ the result is trivial. Suppose next that $k=1$ and $X=\{x\}$. Since $f_{m}(\{x\},\{y\})=w_{m}^{\mathrm{SAW}}(x, y)$, we have $\sum_{m=0}^{\infty} \Lambda^{-m} f_{m}(\{x\},\{y\}) \leqslant 1$ for each $y \in Y$ by Proposition 5.3. Thus $\sum_{m=0}^{\infty} \Lambda^{-m} f_{m}(\{x\}, Y) \leqslant|Y|$ and the proposition holds for $k=1$.

Suppose now that $k \geqslant 2$. Let $x$ be any vertex in $X$; by assumption $x \notin Y$. By Lemma 6.5, given any $F \in \mathcal{F}_{m}(X, Y)$, we can decompose $F$ into a forest $F_{1} \in \mathcal{F}_{i}(X \backslash x, Y)$ and a path $P \in \mathcal{W}_{m-i}^{\text {FPSAW }}\left(x, V\left(F_{1}\right)\right)$; and each such pair $\left(F_{1}, P\right)$ arises from a unique $F$. Since $w(F)=w\left(F_{1}\right) w(P)$, we have

$$
\begin{aligned}
& \sum_{m=0}^{\infty}(m+|Y|)^{-(k-1)} \Lambda^{-m} \sum_{F \in \mathcal{F}_{m}(X, Y)} w(F) \\
& \quad=\sum_{m=0}^{\infty}(m+|Y|)^{-(k-1)} \sum_{i=0}^{m} \Lambda^{-i} \sum_{F_{1} \in \mathcal{F}_{i}(X \backslash x, Y)} w\left(F_{1}\right) \Lambda^{-(m-i)} \sum_{P \in \mathcal{W}_{m-i}^{\text {FPSAW }}\left(x, V\left(F_{1}\right)\right)} w(P) \\
& \quad=\sum_{i=0}^{\infty} \Lambda^{-i} \sum_{F_{1} \in \mathcal{F}_{i}(X \backslash x, Y)} w\left(F_{1}\right) \sum_{j=0}^{\infty} \Lambda^{-j}(i+j+|Y|)^{-(k-1)} \sum_{P \in \mathcal{W}_{j}^{\text {FPSAW }}\left(x, V\left(F_{1}\right)\right)} w(P) .
\end{aligned}
$$


Now, for each fixed $F_{1}$ we have $\sum_{j=0}^{\infty} \Lambda^{-j} \sum_{P \in \mathcal{W}_{j}^{\mathrm{FPSAW}}\left(x, V\left(F_{1}\right)\right)} w(P) \leqslant\left|V\left(F_{1}\right)\right|$ by the base case $k=1$. Since $\left|V\left(F_{1}\right)\right|=i+|Y| \leqslant i+j+|Y|$, we have

$$
\begin{aligned}
\sum_{m=0}^{\infty}(m+|Y|)^{-(k-1)} \Lambda^{-m} \sum_{F \in \mathcal{F}_{m}(X, Y)} w(F) & \leqslant \sum_{i=0}^{\infty}(i+|Y|)^{-(k-2)} \Lambda^{-i} \sum_{F_{1} \in \mathcal{F}_{i}(X \backslash x, Y)} w\left(F_{1}\right) \\
& \leqslant 1
\end{aligned}
$$

by the inductive hypothesis.

When $|X|=1$, Proposition 6.2 is in some sense best possible. To see this, take $G$ to be a star, $X$ to be the central vertex and $Y$ to be the end-vertices: this gives $\Lambda=1$, $f_{1}(X, Y)=|Y|$ and $f_{m}(X, Y)=0$ for $m \neq 1$, so that $\sum_{m=0}^{\infty} \Lambda^{-m} f_{m}(X, Y)=|Y|$. When $|X|=k \geqslant 2$, by contrast, Proposition 6.2 is perhaps not best possible. If we take $G$ to be the disjoint union of $k$ isomorphic stars (again with central vertices in $X$ and endvertices in $Y$ ), we have $\sum_{m=0}^{\infty} \Lambda^{-m} f_{m}(X, Y)=(|Y| / k)^{k}$. This shows that if there is a universal upper bound on $\sum_{m=0}^{\infty} \Lambda^{-m} f_{m}(X, Y)$, the right-hand side has to grow at least like $(|Y| /|X|)^{|X|}$. We suspect the following conjectures are true:

Conjecture 6.6 For all $X, Y \subseteq V(G)$ with $Y \neq \varnothing$, we have

$$
\sum_{m=0}^{\infty} \Lambda^{-m} f_{m}(X, Y) \leqslant|Y|^{|X|} .
$$

Conjecture 6.7 (a special case of Conjecture 6.6) For all nonempty $X \subseteq V(G)$, we have

$$
\sum_{m=0}^{\infty} \Lambda^{-m} t_{m}(X) \leqslant 1 .
$$

\subsection{The class $\mathcal{H}_{m}(X)$}

We conclude this section by discussing a larger class of forests. For $X \subseteq V(G)$, let $\mathcal{H}_{m}(X)$ be the set of all $m$-edge forests $F$ in $G$ such that $L(F) \subseteq X \subseteq V(F)$. For integers $p, r \geqslant 1$, let $\mathcal{H}_{m}(X, p)$ be the set of all $m$-edge forests $F$ in $\mathcal{H}_{m}(X)$ such that each component of $F$ contains at least $p$ vertices of $X$, and let $\mathcal{H}_{m}(X, p, r)$ be the set of all forests $F$ in $\mathcal{H}_{m}(X, p)$ such that $F$ has precisely $r$ components. Put

$$
h_{m}(X)=\sum_{F \in \mathcal{H}_{m}(X)} w(F)
$$

and define $h_{m}(X, p)$ and $h_{m}(X, p, r)$ similarly. Our next result uses Proposition 6.1 to bound $h_{m}(X, p, r)$ in terms of $\Delta$ : 
Proposition 6.8 Let $X \subseteq V(G)$ where $|X|=k \geqslant$ rp. Then

$$
\sum_{m=0}^{\infty} \Delta^{-m} h_{m}(X, p, r) \leqslant p^{-(r-1)}(k-r p+p)^{-1}\left(\begin{array}{l}
k \\
r
\end{array}\right) \text {. }
$$

Proof. Choose $F \in \mathcal{H}_{m}(X, p, r)$. Let $\left\{X_{1}, X_{2}, \ldots, X_{r}\right\}$ be the partition of $X$ determined by the components of $F$. Then $F \in \mathcal{F}_{m}(X, Y)$ for all sets $Y$ such that $\left|Y \cap X_{j}\right|=1$ for all $1 \leqslant j \leqslant r$. Hence there are precisely $\prod_{j=1}^{r}\left|X_{j}\right|$ different sets $Y \subseteq X$ such that $F \in \mathcal{F}_{m}(X, Y)$. Since $\left|X_{j}\right| \geqslant p$ for all $j(1 \leqslant j \leqslant r)$ and $\sum_{j=1}^{r}\left|X_{j}\right|=k$, it follows that $\prod_{j=1}^{r}\left|X_{j}\right| \geqslant p^{r-1}(k-r p+p)$. Thus

$$
\sum_{F \in \mathcal{H}_{m}(X, p, r)} w(F) \leqslant p^{-(r-1)}(k-r p+p)^{-1} \sum_{\substack{Y \subseteq X \\|Y|=r}} \sum_{F \in \mathcal{F}_{m}(X, Y)} w(F) .
$$

Using Proposition 6.1 we deduce

$$
\begin{aligned}
\sum_{m=0}^{\infty} \sum_{F \in \mathcal{H}_{m}(X, p, r)} \Delta^{-m} w(F) & \leqslant p^{-(r-1)}(k-r p+p)^{-1} \sum_{m=0}^{\infty} \Delta^{-m} \sum_{\substack{Y \subseteq X \\
|Y|=r}} \sum_{F \in \mathcal{F}_{m}(X, Y)} w(F) \\
& \leqslant p^{-(r-1)}(k-r p+p)^{-1}\left(\begin{array}{c}
k \\
r
\end{array}\right) .
\end{aligned}
$$

Summing over $r$, we obtain:

Corollary 6.9 Let $X \subseteq V(G)$ where $|X|=k \geqslant 1$. Then

$$
\begin{aligned}
\sum_{m=0}^{\infty} \Delta^{-m} h_{m}(X, p) & \leqslant \sum_{r=1}^{\lfloor k / p\rfloor} p^{-(r-1)}(k-r p+p)^{-1}\left(\begin{array}{l}
k \\
r
\end{array}\right) \\
& \leqslant\left(1+\frac{1}{p}\right)^{k}-1
\end{aligned}
$$

Here the crude upper bound (6.17b) is obtained from $(6.17 \mathrm{a})$ by replacing $(k-r p+p)^{-1}$ by $p^{-1}$. The true large- $k$ asymptotic behavior of $(6.17 \mathrm{a})$ is $(1+1 / p)^{k} k^{-1} p(p+1)[1+O(1 / k)]$.

Taking $p=1$ in (6.17a) gives:

Corollary 6.10 Let $X \subseteq V(G)$ where $|X|=k \geqslant 1$. Then

$$
\sum_{m=0}^{\infty} \Delta^{-m} h_{m}(X) \leqslant \frac{2\left(2^{k}-1\right)}{k+1}
$$


Using a similar proof technique to that of Proposition 6.8, but using Proposition 6.2 instead of Proposition 6.1, we may deduce

Proposition 6.11 Let $X \subseteq V(G)$ where $|X|=k \geqslant$ rp. Then

$$
\sum_{m=0}^{\infty}(m+r)^{-(k-1)} \Lambda^{-m} h_{m}(X, p, r) \leqslant r p^{-(r-1)}(k-r p+p)^{-1}\left(\begin{array}{l}
k \\
r
\end{array}\right) .
$$

\section{Counting Connected Subgraphs (and Related Ob- jects)}

For $X \subseteq V(G)$, let $\mathcal{C}_{m}(X)$ be the set of all $m$-edge subgraphs $H$ in $G$ (connected or not) such that

(C1) $X \subseteq V(H)$, and

(C2) each component of $H$ contains at least one vertex in $X$.

Note in particular the following special cases:

- For any $X, \mathcal{C}_{0}(X)$ has as its single element the edgeless graph with vertex set $X$.

- $\mathcal{C}_{0}(\varnothing)$ has as its single element the empty graph, and $\mathcal{C}_{m}(\varnothing)=\varnothing$ for $m \geqslant 1$.

- $\mathcal{C}_{m}(\{x\})$ consists of the connected $m$-edge subgraphs that contain $x$.

Recall the definition $w(H)=\prod_{e \in E(H)} w_{e}$. Define the weighted counts

$$
c_{m}(X)=\sum_{H \in \mathcal{C}_{m}(X)} w(H)
$$

We then have the following bound in terms of maximum weighted degree:

Proposition 7.1 Whenever $|X|=k \geqslant 0$ we have

$$
c_{m}(X) \leqslant C(m, k) \Delta^{m}
$$

where

$$
C(m, k)= \begin{cases}k(m+k)^{m-1} / m ! & \text { for } k \neq 0 \\ \delta_{m 0} & \text { for } k=0\end{cases}
$$

The $k=1$ case of Proposition 7.1 was proven previously [14, Proposition 4.5].

Before beginning the proof of Proposition 7.1, let us note some facts about the numbers $C(m, k)$ :

(a) For each integer $m \geqslant 0, C(m, k)$ is a polynomial of degree $m$ in $k$. 
(b) For each integer $m \geqslant 0, C(m, k)$ is an increasing function of $k$ for $k \geqslant 0$.

(c) Generating function: If $\mathrm{C}(z)$ solves the equation

$$
\mathrm{C}(z)=e^{z \mathrm{C}(z)}
$$

then

$$
\mathrm{C}(z)^{k}=\sum_{m=0}^{\infty} C(m, k) z^{m}
$$

for all $k$ (integer or not); this follows from the Lagrange inversion formula. ${ }^{7}$ Moreover, the series (7.5) is absolutely convergent for $|z| \leqslant 1 / e$ and satisfies $\mathrm{C}(1 / e)=e$.

(d) For integer $k \geqslant 1$,

$$
C(m, k)=\sum_{\substack{m_{1}, \ldots, m_{k} \geqslant 0 \\ m_{1}+\cdots+m_{k}=m}} \prod_{i=1}^{k} C\left(m_{i}, 1\right) .
$$

This is an immediate consequence of (7.5).

(e) For all $k$ and $z$ (integer or not),

$$
C(m, k)=\sum_{f=0}^{m} \frac{z^{f}}{f !} C(m-f, k-z+f) .
$$

This can easily be verified by direct calculation using the binomial formula:

$$
\begin{aligned}
& \sum_{f=0}^{m} \frac{z^{f}}{f !} \frac{(k-z+f)(m+k-z)^{m-f-1}}{(m-f) !} \\
& \quad=\frac{1}{m !} \sum_{f=0}^{m}\left(\begin{array}{c}
m \\
f
\end{array}\right)(k-z+f) z^{f}(m+k-z)^{m-f-1}
\end{aligned}
$$

${ }^{7}$ Proof. We use the Lagrange inversion formula in the form [16, Theorem 5.4.2]:

Let $G(z)=\sum_{n=0}^{\infty} a_{n} z^{n}$ be a formal power series with $a_{0} \neq 0$. Then there is a unique formal power series $f(z)=\sum_{n=1}^{\infty} b_{n} z^{n}$ satisfying $f(z)=z G(f(z))$, and it satisfies

$$
\left[z^{n}\right] f(z)^{k}=\frac{k}{n}\left[z^{n-k}\right] G(z)^{n},
$$

where $\left[z^{m}\right] F(z)$ denotes the coefficient of $z^{m}$ in the formal power series $F(z)$.

Now, equation (7.4) multiplied by $z$ has the given form with $f(z)=z \mathrm{C}(z)$ and $G=$ exp. It follows that

$$
\left[z^{n-k}\right] \mathrm{C}(z)^{k}=\frac{k}{n}\left[z^{n-k}\right] e^{n z}=\frac{k}{n} \frac{n^{n-k}}{(n-k) !},
$$

which upon setting $n=m+k$ gives $\left[z^{m}\right] \mathrm{C}(z)^{k}=k(m+k)^{m-1} / m !$. 


$$
\begin{aligned}
& =\frac{1}{m !}\left(1-\frac{d}{d k}\right) \sum_{f=0}^{m}\left(\begin{array}{c}
m \\
f
\end{array}\right) z^{f}(m+k-z)^{m-f} \\
& =\frac{1}{m !}\left(1-\frac{d}{d k}\right)(m+k)^{m} \\
& =\frac{1}{m !}\left[(m+k)^{m}-m(m+k)^{m-1}\right] \\
& =\frac{k(m+k)^{m-1}}{m !} .
\end{aligned}
$$

(f) For each fixed $k>0$, we have

$$
C(m, k)=e^{m} m^{-3 / 2} \frac{k e^{k}}{\sqrt{2 \pi}}[1+O(1 / m)]
$$

as $m \rightarrow \infty$. This is an immediate consequence of Stirling's formula.

In proving Proposition 7.1, we will use the following two facts about the weighted counts $c_{m}(X)$ for $X=\left\{x_{1}, x_{2}, \ldots, x_{k}\right\} \subseteq V(G)$ where $x_{1}, x_{2}, \ldots, x_{k}$ are all distinct:

Fact 1. $c_{m}(X) \leqslant \sum_{\substack{m_{1}, \ldots, m_{k} \geqslant 0 \\ m_{1}+\cdots+m_{k}=m}} \prod_{i=1}^{k} c_{m_{i}}\left(x_{i}\right)$.

Proof. Trivial when $k=1$. For $k \geqslant 2$, construct a weight-preserving bijection $\mathbf{F}$ of $\mathcal{C}_{m}(X)$ onto a subset of $\underset{\begin{array}{c}m_{1}, \ldots, m_{k} \geqslant 0 \\ m_{1}+\cdots+m_{k}=m\end{array}}{\bigcup} \prod_{i=1}^{k} \mathcal{C}_{m_{i}}\left(x_{i}\right)$ as follows: Given $H \in \mathcal{C}_{m}(X)$, define $\mathbf{F}(H)_{i}$ to be the component of $H$ containing $x_{i}$ if this component contains no vertex $x_{i^{\prime}}$ with $i^{\prime}<i$, and the graph with vertex set $\left\{x_{i}\right\}$ and no edges, otherwise.

Fact 2. $c_{m}(X) \leqslant \sum_{F \subseteq C\left(x_{1}\right)} w(F) c_{m-|F|}\left(\left(X-x_{1}\right) \cup Y^{F}\right)$ where $C\left(x_{1}\right)$ is shorthand for the cocycle $E\left(\left\{x_{1}\right\},\left\{x_{1}\right\}^{c}\right)$, and $Y^{F}$ denotes the set of endpoints other than $x_{1}$ of the edges in $F$. [Of course, the sum can be limited to sets $F$ having $|F| \leqslant m$, since $c_{m}(X)=0$ for $m<0$.]

Proof. We classify the subgraphs $H \in \mathcal{C}_{m}(X)$ according to $F \equiv E(H) \cap C\left(x_{1}\right)$. So, for each $F \subseteq C\left(x_{1}\right)$, let $\mathcal{C}_{m}(X ; F)$ be the set of all $H \in \mathcal{C}_{m}(X)$ that have $E(H) \cap C\left(x_{1}\right)=F$. For $H \in \mathcal{C}_{m}(X ; F)$, we let $H^{\prime}=H \backslash x_{1}$. Then $\left|E\left(H^{\prime}\right)\right|=m-|F|$ and $H^{\prime} \in \mathcal{C}_{m-|F|}((X-$ $\left.\left.x_{1}\right) \cup Y^{F}\right)$. This gives an injective map from $\mathcal{C}_{m}(X ; F)$ into $\mathcal{C}_{m-|F|}\left(\left(X-x_{1}\right) \cup Y^{F}\right)$. Fact 2 now follows since $w(H)=w(F) w\left(H^{\prime}\right)$.

We now give two alternative proofs of Proposition 7.1. The first proof uses Fact 2 for $k=1$ only, together with Fact 1; it leads to a nonlinear recursion whose solution is 
$C(m, 1)$, namely $(7.7)$ with $k=z=1$ combined with (7.6). The second proof uses Fact 2 for all $k$, but does not use Fact 1; it leads to a linear recursion whose solution is $C(m, k)$, namely (7.7) with $z=1$.

We will need the following elementary result:

Lemma 7.2 Let $S$ be a set in which each element $e \in S$ is given a nonnegative real weight $w_{e}$. Then, for each integer $f \geqslant 0$, we have

$$
\sum_{\substack{F \subseteq S \\|F|=f}} \prod_{e \in F} w_{e} \leqslant \frac{1}{f !}\left(\sum_{e \in S} w_{e}\right)^{f}
$$

First proof of Proposition 7.1. Consider first the case $k=1$, hence $X=\{x\}$. We use induction on $m$. The proposition holds trivially when $m=0$, so let us assume that $m \geqslant 1$. We apply Fact 2 with $k=1$, and observe that the term $F=\varnothing$ contributes zero when $m \geqslant 1\left[\right.$ since $\left.c_{m-|F|}\left(Y^{F}\right)=c_{m}(\varnothing)=0\right]$; we therefore have

$$
c_{m}(x) \leqslant \sum_{\substack{\varnothing \neq F \subseteq C(x) \\|F| \leqslant m}} w(F) c_{m-|F|}\left(Y^{F}\right) .
$$

Applying Fact 1 to bound $c_{m-|F|}\left(Y^{F}\right)$, we obtain

$$
c_{m}(x) \leqslant \sum_{\substack{\varnothing \neq F \subseteq C(x) \\|F| \leqslant m}} w(F) \sum_{\substack{m_{1}, \ldots, m_{|Y F|} \geqslant 0 \\ m_{1}+\cdots+m_{|Y F|}=m-|F|}} \prod_{i=1}^{\mid Y^{F \mid}} c_{m_{i}}\left(v_{i}\right)
$$

where $Y^{F}=\left\{v_{1}, \ldots, v_{\left|Y^{F}\right|}\right\}$. Note that we have $m_{i}<m$ for all $i$ since $|F| \geqslant 1$. We can therefore apply the inductive hypothesis $c_{m_{i}}\left(v_{i}\right) \leqslant C\left(m_{i}, 1\right) \Delta^{m_{i}}$ to obtain

$$
\begin{aligned}
c_{m}(x) \leqslant \sum_{\substack{\varnothing \neq F \subseteq C(x) \\
|F| \leqslant m}} w(F) \sum_{\substack{m_{1}, \ldots, m_{|Y F|} \geqslant 0 \\
m_{1}+\cdots+m_{|Y F|}=m-|F|}} \prod_{i=1}^{\left|Y^{F}\right|} C\left(m_{i}, 1\right) \Delta^{m_{i}} \\
=\sum_{\substack{\varnothing \neq F \subseteq C(x) \\
|F| \leqslant m}} w(F) C\left(m-|F|,\left|Y^{F}\right|\right) \Delta^{m-|F|} \\
\leqslant \sum_{\substack{\varnothing \neq F \subseteq C(x) \\
|F| \leqslant m}} w(F) C(m-|F|,|F|) \Delta^{m-|F|}
\end{aligned}
$$


where the second line used the identity (7.6), and the last step used $\left|Y^{F}\right| \leqslant|F|$ and the fact that $C(m, k)$ is an increasing function of $k .^{8}$ Using Lemma 7.2, we have

$$
\begin{aligned}
c_{m}(x) & \leqslant \sum_{f=1}^{m} \frac{\Delta^{f}}{f !} C(m-f, f) \Delta^{m-f} \\
& =\sum_{f=0}^{m} \frac{\Delta^{f}}{f !} C(m-f, f) \Delta^{m-f} \\
& =C(m, 1) \Delta^{m}
\end{aligned}
$$

where the second line used $C(m, 0)=0$ for $m \geqslant 1$, and the last line used identity (7.7) with $k=z=1$. This proves Proposition 7.1 for $k=1$.

The result for general $k$ now follows using Fact 1 and the identity (7.6).

Second proof of Proposition 7.1. We use induction on $m+k$. The proposition holds trivially when $m=0$, so we assume $m \geqslant 1$. Apply Fact 2 for general $k$; because the right-hand side involves quantities $c_{m-|F|}\left(\left(X-x_{1}\right) \cup Y^{F}\right)$ with $m-|F|+\left|\left(X-x_{1}\right) \cup Y^{F}\right| \leqslant$ $m+k-1$, we can apply the inductive hypothesis $c_{m-|F|}\left(\left(X-x_{1}\right) \cup Y^{F}\right) \leqslant C(m-|F|, \mid(X-$ $\left.\left.x_{1}\right) \cup Y^{F} \mid\right) \Delta^{m-|F|}$ to conclude that

$$
\begin{aligned}
c_{m}(X) & \leqslant \sum_{F \subseteq C\left(x_{1}\right)} w(F) C\left(m-|F|,\left|\left(X-x_{1}\right) \cup Y^{F}\right|\right) \Delta^{m-|F|} \\
& \leqslant \sum_{F \subseteq C\left(x_{1}\right)} w(F) C(m-|F|, k-1+|F|) \Delta^{m-|F|} \\
& \leqslant \sum_{f=0}^{m} \frac{\Delta^{f}}{f !} C(m-f, k-1+f) \Delta^{m-f} \\
& =C(m, k) \Delta^{m},
\end{aligned}
$$

where the second line used $\left|\left(X-x_{1}\right) \cup Y^{F}\right| \leqslant k-1+\left|Y^{F}\right| \leqslant k-1+|F|$ and the fact that $C(m, k)$ is increasing with $k$, the third line used Lemma 7.2, and the last line used identity (7.7) with $z=1$.

Let us now show that Proposition 7.1 is best possible:

Example 7.1. Let $\mathbf{T}_{r}$ be the infinite $r$-regular tree, let $x_{1}, \ldots, x_{k} \in V\left(\mathbf{T}_{r}\right)$ satisfy $\operatorname{dist}\left(x_{i}, x_{j}\right)>m$ for $i \neq j$, and let $G$ be the subtree of $\mathbf{T}_{r}$ induced by the vertices lying at a distance at most $M$ from the set $\left\{x_{1}, \ldots, x_{k}\right\}$, where $M$ is any fixed number greater

\footnotetext{
${ }^{8}$ If $G$ is a simple graph, we have $\left|Y^{F}\right|=|F|$. But if $G$ has multiple edges, all we can say is that $\left|Y^{F}\right| \leqslant|F|$.
} 
than or equal to $m$. Let all edge weights $w_{e}$ equal $\Delta / r$. Then by a slight generalization of the computation in [14, proof of Proposition 4.2], one shows that

$$
c_{m}\left(x_{1}, \ldots, x_{k}\right)=\left(\frac{\Delta}{r}\right)^{m} \frac{k r}{m}\left(\begin{array}{c}
k r+(r-1) m-1 \\
m-1
\end{array}\right),
$$

which in the limit $r \rightarrow \infty$ with $\Delta$ fixed tends to $C(m, k) \Delta^{m}$.

Example 7.2. Let $G$ be the complete graph $K_{n}$, and let all edge weights $w_{e}$ equal $\Delta /(n-1)$. Consider first the case $k=1$ : let us count the subset of $\mathcal{C}_{m}(\{x\})$ consisting of the $m$-edge trees containing $x$. The number of such trees is $\left(\begin{array}{c}n-1 \\ m\end{array}\right)(m+1)^{m-1}$, where the first factor counts the number of ways we can choose $m$ additional vertices and the second factor counts the number of trees on $m+1$ labelled vertices. We therefore have

$$
c_{m}(x) \geqslant\left(\begin{array}{c}
n-1 \\
m
\end{array}\right)(m+1)^{m-1}\left(\frac{\Delta}{n-1}\right)^{m},
$$

which in the limit $n \rightarrow \infty$ with $m$ fixed tends to $C(m, 1) \Delta^{m}$.

Now consider general $k$ : let $x_{1}, \ldots, x_{k}$ be distinct vertices, and let us count the subset of $\mathcal{C}_{m}\left(x_{1}, \ldots, x_{k}\right)$ consisting of the $m$-edge $k$-component forests in which each component contains exactly one $x_{i}$. By a similar counting argument we have

$$
\begin{aligned}
& c_{m}\left(x_{1}, \ldots, x_{k}\right) \geqslant \\
& \left(\frac{\Delta}{n-1}\right)^{m} \sum_{\substack{m_{1}, \ldots, m_{k} \geqslant 0 \\
m_{1}+\cdots+m_{k}=m}} \prod_{i=1}^{k}\left(\begin{array}{c}
n-k-m_{1}-\ldots-m_{i-1} \\
m_{i}
\end{array}\right)\left(m_{i}+1\right)^{m_{i}-1},
\end{aligned}
$$

which in the limit $n \rightarrow \infty$ with $m$ fixed tends to $C(m, k) \Delta^{m}[$ using (7.6)].

Note the difference between the "pointwise" bounds of Propositions 5.1 and 7.1 and the "generating-function" bounds of Propositions 5.2, 5.3, 6.1 and 6.2. In the former cases, the bound can be saturated simultaneously for all (or arbitrarily many) $m$ - as shown by the preceding two examples - so nothing can be gained by summing over $m$. In the latter cases, by contrast, the pointwise bound can be saturated only for one $m$ at a time (cf. Example 5.3 after Corollary 5.5), so the generating-function bound is stronger. The fundamental difference between the two situations is that the subgraphs considered in Propositions 5.1 and 7.1 are "tied down only at one end" (and hence can grow freely in all directions), while those in Propositions 5.2, 5.3, 6.1 and 6.2 are "tied down at all the leaves".

\section{Counting Blocks, Block Paths, Block Trees and Block Forests}

We now seek an analogue of Proposition 7.1 using maxmaxflow in place of maximum degree. Unfortunately, no such bound is possible for the families $\mathcal{C}_{m}(X)$ : a simple 
counterexample is provided by the stars $G=K_{1, r}$, which have maximum degree $r$ but maxmaxflow 1 (independent of $r$ ); letting $x$ be the central vertex of the star, we have $\left|\mathcal{C}_{1}(\{x\})\right|=r$, which is unbounded as $r \rightarrow \infty$. The same thing happens for wheels $G=K_{1}+C_{r}$, so it is no help to assume that $G$ is non-separable.

To get a bound in terms of maxmaxflow, we need to restrict attention to a suitably chosen proper subfamily of $\mathcal{C}_{m}(X)$ : roughly speaking, we need to count subgraphs $H$ that possess suitable "non-separability properties". After much experimentation, we settled on the family $\mathcal{B T}_{m}(X)$ of "block trees" and the families $\mathcal{B F}_{m}(X, Y)$ and $\mathcal{B F}_{m}^{*}(X, Y)$ of "block forests", to be defined in Section 8.1. However, simpler but weaker results can be obtained using a different (and slightly larger) family $\mathcal{B}_{m}(X)$ of block forests, to be defined in Section 8.2. These two subsections are independent of each other and can be read in either order.

\subsection{The classes $\mathcal{B T}_{m}(X), \mathcal{B F}_{m}(X, Y)$ and $\mathcal{B F}_{m}^{*}(X, Y)$}

Let $H$ be a not-necessarily-connected graph. We recall that a vertex of $H$ is called an internal vertex of $H$ if it is not a cut vertex of $H$. Now let $B$ be a block of $H$. We denote by $\operatorname{Int}(B, H)$ the set of internal vertices of $H$ that belong to $B$. We say that $B$ is an isolated block of $H$ if it contains no cut vertices of $H$, and an end block of $H$ if it contains exactly one cut vertex of $H$. Finally, if $x, y \in V(H)$ with $x \neq y$, we say that $H$ is an $x y$-block path if $H$ is connected and is either a block (hence an isolated block of itself) or else has exactly two end blocks $B_{1}, B_{2}$ with $x \in \operatorname{Int}\left(B_{1}, H\right)$ and $y \in \operatorname{Int}\left(B_{2}, H\right)$.

For any nonempty $X \subseteq V(G)$, let $\mathcal{B T}_{m}(X)$ be the set of all $m$-edge subgraphs $H$ in $G$ such that

(BT1) $X \subseteq V(H)$;

(BT2) $H$ is connected;

(BT3) each end block $B$ of $H$ contains at least one vertex of $X$ as an internal vertex [that is, $\operatorname{Int}(B, H) \cap X \neq \varnothing]$; and

(BT4) if $H$ is a block, then either $H$ is an isolated vertex [hence $V(H)=X=$ $\{x\}$ ] or else $H$ contains at least two vertices of $X$.

By analogy with the set $\mathcal{T}_{m}(X)$, which consists of trees whose leaves are "tied down" on the set $X$, we think of the elements of $\mathcal{B T}_{m}(X)$ as block trees whose end blocks are "tied down" on the set $X$. [In particular, we have $\mathcal{T}_{m}(X) \subseteq \mathcal{B} \mathcal{T}_{m}(X)$.] Note the following special cases:

- $\mathcal{B T}_{0}(\{x\})$ has as its single element the edgeless graph with vertex set $\{x\}$, and $\mathcal{B T}_{m}(\{x\})=\varnothing$ for $m \geqslant 1$.

- When $x \neq y, \mathcal{B T}_{m}(\{x, y\})$ is the set of $m$-edge $x y$-block paths.

- $\mathcal{B T}_{0}(X)=\varnothing$ for $|X| \geqslant 2$. 
For $X, Y \subseteq V(G)$ with $Y \neq \varnothing$, let $\mathcal{B F}_{m}(X, Y)$ be the set of all $m$-edge subgraphs $H$ in $G$ (connected or not) such that

(BF1) $X \cup Y \subseteq V(H)$;

(BF2) each component of $H$ contains exactly one vertex of $Y$;

(BF3) each end block $B$ of $H$ contains at least one vertex of $X \cup Y$ as an internal vertex [that is, $\operatorname{Int}(B, H) \cap(X \cup Y) \neq \varnothing]$; and

(BF4) each isolated block of $H$ is either an isolated vertex belonging to $Y$ or else contains at least two vertices of $X \cup Y$.

[Note that $\mathcal{B F}_{m}(X, Y)=\mathcal{B F}_{m}(X \backslash Y, Y)$, so we can assume without loss of generality, if desired, that $X \cap Y=\varnothing$.] By analogy with the set $\mathcal{F}_{m}(X, Y)$, which consists of forests whose leaves are "tied down" on the set $X \cup Y$ and whose components are "tied down" on single elements of the set $Y$, we think of the elements of $\mathcal{B F}_{m}(X, Y)$ as block forests whose end blocks are "tied down" on the set $X \cup Y$ and whose components are "tied down" on single elements of the set $Y$. [In particular, we have $\mathcal{F}_{m}(X, Y) \subseteq \mathcal{B F}_{m}(X, Y) \subseteq \mathcal{C}_{m}(Y)$.] Note the following special cases:

- If $m=0$, then $\mathcal{B F}_{0}(X, Y)=\varnothing$ whenever $X \nsubseteq Y Y$, and $\mathcal{B F}_{0}(X, Y)$ has as its single element the edgeless graph with vertex set $Y$ whenever $X \subseteq Y$.

- If $X=\varnothing$ (or more generally if $X \subseteq Y$ ), then $\mathcal{B F}_{0}(X, Y)$ has as its single element the edgeless graph with vertex set $Y$, and $\mathcal{B F}_{m}(X, Y)=\varnothing$ for $m \geqslant 1$.

- If $X=\{x\}$ with $x \notin Y$, then each $H \in \mathcal{B F}_{m}(\{x\}, Y)$ is the disjoint union of an $m$-edge $x y$-block path for some $y \in Y$ (this component avoiding the set $Y \backslash y$ ) and the collection $Y \backslash y$ of isolated vertices.

- For $Y=\{y\}$, we have $\mathcal{B F}_{m}(X,\{y\})=\mathcal{B T}_{m}(X \cup\{y\})$.

Recall that $w(H)=\prod_{e \in E(H)} w_{e}$. Define the weighted counts

$$
\begin{aligned}
b t_{m}(X) & =\sum_{H \in \mathcal{B \mathcal { T }}_{m}(X)} w(H) \\
b f_{m}(X, Y) & =\sum_{H \in \mathcal{B F}_{m}(X, Y)} w(H)
\end{aligned}
$$

We will obtain the following bounds on $b f_{m}(X, Y)$ in terms of $\Delta$ and $\Lambda$ :

Proposition 8.1 For all $X, Y \subseteq V(G)$ with $Y \neq \varnothing$, we have

$$
\sum_{m=0}^{\infty}\left(\frac{\Delta}{\ln 2}\right)^{-m} b f_{m}(X, Y) \leqslant 1 .
$$


Proposition 8.2 For all $X, Y \subseteq V(G)$ with $Y \neq \varnothing$ and all $\alpha \in(1,2]$, we have

$$
\sum_{m=0}^{\infty}\left(\frac{\alpha \Lambda}{\ln \alpha}\right)^{-m} b f_{m}(X, Y) \leqslant \alpha^{|Y|-1} .
$$

Using the identity $\mathcal{B F}_{m}(X,\{y\})=\mathcal{B T}_{m}(X \cup\{y\})$, we immediately obtain the following corollaries for block trees:

Corollary 8.3 For all nonempty $X \subseteq V(G)$, we have

$$
\sum_{m=0}^{\infty}\left(\frac{\Delta}{\ln 2}\right)^{-m} b t_{m}(X) \leqslant 1 .
$$

Corollary 8.4 For all nonempty $X \subseteq V(G)$, we have

$$
\sum_{m=0}^{\infty}\left(\frac{2 \Lambda}{\ln 2}\right)^{-m} b t_{m}(X) \leqslant 1 .
$$

Corollary 8.3 could be proved directly using the same proof technique as for Proposition 8.1. It is curious to note, however, that we have been unable to find a direct proof of Corollary 8.4; our proof of Proposition 8.2 employs an inner induction on $|Y|$, and thus inevitably passes through disconnected graphs.

As an immediate consequence of Corollary 8.4, we obtain:

Corollary 8.5 Fix a weighted graph $(G, \mathbf{w})$ and an edge $e \in E(G)$. Let $b_{m}(e)$ be the sum of the weights of the non-separable m-edge subgraphs of $G$ containing $e$. Then $b_{1}(e)=w_{e}$ and

$$
\sum_{m=2}^{\infty}\left(\frac{2 \Lambda(G-e, \mathbf{w})}{\ln 2}\right)^{-(m-1)} b_{m}(e) \leqslant w_{e} .
$$

Proof of Corollary 8.5, assuming Corollary 8.4. Let $e=x y$ and put $X=$ $\{x, y\}$. Clearly $b_{1}(e)=w_{e}$. Let $H$ be a subgraph of $G$ and let $m \geqslant 2$. Then $H$ is a non-separable $m$-edge subgraph of $G$ containing $e$ if and only if $H-e \in \mathcal{B T}_{m-1}(X)$. Thus Corollary 8.4 applied to $G-e$ gives

$$
\begin{aligned}
\sum_{m=2}^{\infty}\left(\frac{2 \Lambda(G-e, \mathbf{w})}{\ln 2}\right)^{-(m-1)} b_{m}(e) & =w_{e} \sum_{m=2}^{\infty}\left(\frac{2 \Lambda(G-e, \mathbf{w})}{\ln 2}\right)^{-(m-1)} b t_{m-1}(X) \\
& \leqslant w_{e}
\end{aligned}
$$

Our proof of Proposition 8.1 combines ideas from the proofs of Propositions 6.1 and 7.1: we use an inner induction on $|X|$ as a substitute for the "point-to-set" bound of 
Proposition 5.2, and we use a "cutting" argument similar to that employed in the proofs of Propositions 5.2 and 7.1 to handle the case $|X|=1$. In the inductive step we shall use the following analogue of Lemma 6.5 to split a block forest with $k$ end blocks into a block forest with $k-1$ end blocks and a block path:

Lemma 8.6 Let $G$ be a graph, let $X, Y \subseteq V(G)$ with $Y \neq \varnothing$, let $x \in X \backslash Y$, and let $H \in \mathcal{B F}_{m}(X, Y)$. Let $H_{1}$ be the convex hull of $(X \backslash x) \cup Y$ in $H$, and let $H_{2}$ be the convex hull of $\{x\} \cup V\left(H_{1}\right)$ in $H \backslash E\left(H_{1}\right)$. Then $H$ is the edge-disjoint union of $H_{1}$ and $H_{2}$; and for some $i(0 \leqslant i \leqslant m)$, we have $H_{1} \in \mathcal{B F}_{i}(X \backslash x, Y)$ and $H_{2} \in \mathcal{B F}_{m-i}\left(\{x\}, V\left(H_{1}\right)\right)$. Moreover, the map $H \mapsto\left(H_{1}, H_{2}\right)$ is an injection.

Note the slight change of perspective from Lemma 6.5: here $H_{2}$ is not an $x y$-block path for some $y \in V\left(H_{1}\right)$, but rather the union of such a block path with the collection $V\left(H_{1}\right) \backslash y$ of isolated vertices. In particular, we have $V\left(H_{1}\right) \subseteq V\left(H_{2}\right)$. However, modulo this change, this decomposition reduces to that of Lemma 6.5 in the special case where $H \in \mathcal{F}_{m}(X, Y)$.

Proof of Proposition 8.1. As noted above, we can assume without loss of generality that $X \cap Y=\varnothing$. Let $c=1 / \ln 2$. We shall show that

$$
\sum_{m=0}^{M}(c \Delta)^{-m} b f_{m}(X, Y) \leqslant 1
$$

for all $M \geqslant 0$, by using an outer induction on $M$ and an inner induction on $|X|$. The base case $M=0$ and $|X|$ arbitrary holds by the first remark after the definition of $\mathcal{B F}_{m}(X, Y)$. The case when $X=\varnothing$ and $M$ is arbitrary holds by the second remark following the definition of $\mathcal{B F}_{m}(X, Y)$. Hence we may suppose that $M \geqslant 1$ and $|X| \geqslant 1$. Our inductive argument consists of two steps:

Step 1. Proof that if (8.9) holds for all $|X|$ and all $M^{\prime}$ with $0 \leqslant M^{\prime}<M$, then it also holds for $|X|=1$ and $M$. This step uses a "cutting" argument.

Step 2. Proof that if (8.9) holds for all $\left|X^{\prime}\right|$ with $1 \leqslant\left|X^{\prime}\right|<|X|$ and some given $M$, then it holds for $|X|$ and the same $M$. This step uses Lemma 8.6.

Step 1. Suppose that (8.9) holds for all $|X|$ and all $M^{\prime}$ with $0 \leqslant M^{\prime}<M$. Now let $X=\{x\}$ (note that $x \notin Y$ by assumption) and consider a subgraph $H \in \mathcal{B F}_{m}(\{x\}, Y)$ for some $m$. Note that $H$ is the disjoint union of an $x y$-block path $H^{\prime}$ for some $y \in Y$ and the collection $Y \backslash y$ of isolated vertices. In particular, $x$ is neither an isolated vertex nor a cut vertex of $H$. Let $F$ be the set of edges of $H$ incident with $x$, let $f=|F|(\geqslant 1$ because $x$ is not isolated), and let $U^{F}$ be the set of end-vertices of edges in $F$ distinct from $x$. Then $H^{\prime}$ remains connected under deletion of $x$ (because $x$ is not a cut vertex).

We shall show that $H \backslash x \in \mathcal{B F}_{m-f}\left(U^{F}, Y\right)$. We have $U^{F} \subseteq V\left(H^{\prime} \backslash x\right)$, so either $H^{\prime} \backslash x=y$ or $H^{\prime} \backslash x$ contains at least two vertices of $U^{F} \cup\{y\}$. For each end block $B$ of 
$H^{\prime} \backslash x$, we have $\operatorname{Int}\left(B, H^{\prime} \backslash x\right) \cap\left(U_{F} \cup\{y\}\right) \neq \varnothing$, otherwise $B$ would be an end block of $H$ with $\operatorname{Int}(B, H) \cap(\{x\} \cup Y)=\varnothing$. Thus $H \backslash x \in \mathcal{B F}_{m-f}\left(U^{F}, Y\right)$.

Let $C$ be the set of edges of $G$ incident to $x$. For each nonempty $F \subseteq C$, let $\mathcal{B F}_{m}(\{x\}, Y ; F)$ be the set of all subgraphs $H \in \mathcal{B F}_{m}(\{x\}, Y)$ such that $E(H) \cap C=F$. Then the map $H \mapsto H \backslash x$ is an injection from $\mathcal{B F}_{m}(\{x\}, Y ; F)$ into $\mathcal{B F}_{m-f}\left(U^{F}, Y\right)$, and $w(H)=w(F) w(H \backslash x)$. Thus

$$
b f_{m}(\{x\}, Y) \leqslant \sum_{\varnothing \neq F \subseteq C} w(F) b f_{m-f}\left(U^{F}, Y\right) .
$$

Hence

$$
\begin{aligned}
\sum_{m=0}^{M}(c \Delta)^{-m} b f_{m}(\{x\}, Y) & =\sum_{m=0}^{M}(c \Delta)^{-m} \sum_{\varnothing \neq F \subseteq C} w(F) b f_{m-f}\left(U^{F}, Y\right) \\
& =\sum_{\varnothing \neq F \subseteq C} w(F)(c \Delta)^{-f} \sum_{j=0}^{M-f}(c \Delta)^{-j} b f_{j}\left(U^{F}, Y\right) \\
& \leqslant \sum_{\varnothing \neq F \subseteq C} w(F)(c \Delta)^{-f}
\end{aligned}
$$

by the outer inductive hypothesis on $M$. Using Lemma 7.2, we deduce that

$$
\begin{aligned}
\sum_{m=0}^{M}(c \Delta)^{-m} b f_{m}(\{x\}, Y) & \leqslant \sum_{f=1}^{\infty}(c \Delta)^{-f} \sum_{\substack{F \subseteq C \\
|F|=f}} w(F) \\
& \leqslant \sum_{f=1}^{\infty}(c \Delta)^{-f} \frac{\Delta^{f}}{f !} \\
& =e^{1 / c}-1=1
\end{aligned}
$$

since $c=1 / \ln 2$. This proves Step 1 .

Step 2. Suppose that (8.9) holds for all $\left|X^{\prime}\right|$ with $1 \leqslant\left|X^{\prime}\right|<|X|$ and some given $M$. Then $|X| \geqslant 2$. Choose arbitrarily some $x \in X$ (recall again that $x \notin Y$ ). Given $H \in$ $\mathcal{B F}_{m}(X, Y)$, by Lemma 8.6 we may decompose $H$ into $H_{1} \cup H_{2}$ where $H_{1} \in \mathcal{B F}_{i}(X \backslash x, Y)$ and $H_{2} \in \mathcal{B F}_{m-i}\left(\{x\}, V\left(H_{1}\right)\right)$. Applying the inductive hypothesis that (8.9) holds both for $X^{\prime}=X \backslash x$ and for $X^{\prime}=\{x\}$ with the given $M$, we may deduce that

$$
\begin{aligned}
& \sum_{m=0}^{M}(c \Delta)^{-m} b f_{m}(X, Y) \\
& \quad=\sum_{m=0}^{M}(c \Delta)^{-m} \sum_{H \in \mathcal{B \mathcal { F }}_{m}(X, Y)} w(H)
\end{aligned}
$$




$$
\begin{aligned}
& \leqslant \sum_{m=0}^{M} \sum_{i=0}^{m}(c \Delta)^{-i} \sum_{H_{1} \in \mathcal{B} \mathcal{F}_{i}(X \backslash x, Y)} w\left(H_{1}\right)(c \Delta)^{-(m-i)} \sum_{H_{2} \in \mathcal{B} \mathcal{F}_{m-i}\left(\{x\}, V\left(H_{1}\right)\right)} w\left(H_{2}\right) \\
& \leqslant \sum_{i=0}^{M}(c \Delta)^{-i} \sum_{H_{1} \in \mathcal{B F}_{i}(X \backslash x, Y)} w\left(H_{1}\right) \sum_{j=0}^{M-i}(c \Delta)^{-j} \sum_{H_{2} \in \mathcal{B F}_{j}\left(\{x\}, V\left(H_{1}\right)\right)} w\left(H_{2}\right) \\
& \leqslant 1 .
\end{aligned}
$$

This completes the proof of Step 2 and hence of the proposition.

Remarks. 1. Step 2 works for any value of $c$. The specific value $c=1 / \ln 2$ enters only in Step 1.

2. Step 2 is "almost" unnecessary; we can "almost" apply Step 1 for any $X$. The trouble is that if $|X|>1$ we might find that $x$ is a cut vertex of $H$. In this case $H \backslash x \notin \mathcal{B F}_{m-f}\left(U^{F}, Y\right)$ since it will have one or more components containing no vertices of $Y$.

Example 8.1. Let $G$ be the graph $K_{2}^{(s)}$ consisting of a pair of vertices $x, y$ joined by $s$ parallel edges. Set all edge weights $w_{e}=\Delta / s$. Let us consider $b t_{m}(\{x, y\})=$ $b f_{m}(\{x\},\{y\})$. The generating function is

$$
\sum_{m=0}^{\infty} \zeta^{m} b f_{m}(\{x\},\{y\})=\left(1+\frac{\Delta}{s} \zeta\right)^{s}-1,
$$

which is an increasing function of $s$ (at fixed $\Delta$ and $\zeta$ ) and tends to $e^{\Delta \zeta}-1$ as $s \rightarrow \infty$. It follows that Proposition 8.1 and Corollary 8.3 are sharp in the sense that $(\ln 2) / \Delta$ is the maximal value of $\zeta$ that allows an upper bound of 1 .

Example 8.2. Let $G$ be the disjoint union of $n$ copies of $K_{2}^{(s)}$; let $X$ contain one vertex from each copy, and let $Y$ be the remaining vertices. Set all edge weights $w_{e}=\Delta / s$. Then

$$
\sum_{m=0}^{\infty} \zeta^{m} b f_{m}(X, Y)=\left[\left(1+\frac{\Delta}{s} \zeta\right)^{s}-1\right]^{n} \stackrel{s \rightarrow \infty}{\longrightarrow}\left(e^{\Delta \zeta}-1\right)^{n} .
$$

So $(\ln 2) / \Delta$ is the maximal value of $\zeta$ that allows any finite upper bound that is independent of $|X|$ and $|Y|$.

Might it be possible to bound $\sum_{m=0}^{\infty} \zeta^{m} b f_{m}(X, Y)$ for some $\zeta>(\ln 2) / \Delta$ if we allow the right-hand side to depend on $|X|$ and $|Y|$ ? We doubt it; but all we can say for sure, at present, is that $\zeta$ cannot exceed $(2 \ln 2) / \Delta$ :

Example 8.3. Let $G$ be the graph $P_{n}^{(s)}$ obtained from the $n$-edge path $(n \geqslant 2)$ by replacing each edge by $s$ parallel edges. Set all edge weights $w_{e}=\Delta /(2 s)$. Let $x, y \in$ $V\left(P_{n}^{(s)}\right)$ with $\operatorname{dist}(x, y)=\ell$. Then

$$
\sum_{m=0}^{\infty} \zeta^{m} b f_{m}(\{x\},\{y\})=\left[\left(1+\frac{\Delta}{2 s} \zeta\right)^{s}-1\right]^{\ell} \stackrel{s \rightarrow \infty}{\longrightarrow}\left(e^{\Delta \zeta / 2}-1\right)^{\ell}
$$


Since $\ell$ can be arbitrarily large, a universal upper bound on $\sum_{m=0}^{\infty} \zeta^{m} b f_{m}(X, Y)$ is impossible for $\zeta>(2 \ln 2) / \Delta$, even when $|X|=|Y|=1$.

We have been unable to obtain a bound for $b f_{m}(X, Y)$ in terms of $\Lambda$ (i.e., Proposition 8.2 or something like it) by extending the proof technique of Proposition 6.2 in a similar way to the above proof. The problem is that a universal "point-to-set" bound of the form $\sum_{m=0}^{\infty}(c \Lambda)^{-m} b f_{m}(X, Y) \leqslant 1$ (with a right-hand side that is independent of $|Y|$ ) is simply not valid for any constant $c$ : it suffices to consider $G=K_{1, r}$ with $r>c$. If, on the other hand, we try to adapt the proof technique of Proposition 8.1 by using an inductive hypothesis of the form $\sum_{m=0}^{\infty}(m+|Y|)^{-(|X|-1)}(c \Lambda)^{-m} b f_{m}(X, Y) \leqslant|Y|$ (similar to that of Proposition 6.2), then we are unable to carry through Step 1 because of the increase in the size of $X$ when $\{x\}$ is replaced by $U^{F}$. Instead, our proof of Proposition 8.2 will rely solely on a "cutting" argument rather than using Lemma 8.6. In order for the induction to go through, we need to work with a slightly larger family of graphs than $\mathcal{B F}_{m}(X, Y)$.

For $X, Y \subseteq V(G)$ with $Y \neq \varnothing$, let $\mathcal{B F}_{m}^{*}(X, Y)$ be the set of all $m$-edge subgraphs $H$ in $G$ (connected or not) such that

(BF1) $X \cup Y \subseteq V(H)$;

(BF2*) each component of $H$ contains at least one vertex of $Y$;

(BF3) each end block $B$ of $H$ contains at least one element of $X \cup Y$ as an internal vertex [that is, $\operatorname{Int}(B, H) \cap(X \cup Y) \neq \varnothing]$; and

(BF4) each isolated block of $H$ is either an isolated vertex belonging to $Y$ or else contains at least two vertices of $X \cup Y$.

The only change from $\mathcal{B F}_{m}(X, Y)$ is, therefore, that each component of $H$ must contain at least one vertex of $Y$, rather than exactly one. We have $\mathcal{B F}_{m}(X, Y) \subseteq \mathcal{B F}_{m}^{*}(X, Y) \subseteq$ $\mathcal{C}_{m}(Y)$. Since $\mathcal{B F}_{m}^{*}(X, Y)=\mathcal{B F}_{m}^{*}(X \backslash Y, Y)$, we can assume without loss of generality, if desired, that $X \cap Y=\varnothing$. Note the special cases:

- If $m=0$, then $\mathcal{B F}_{0}^{*}(X, Y)=\varnothing$ whenever $X \nsubseteq \subseteq Y$, and $\mathcal{B F}_{0}^{*}(X, Y)$ has as its single element the edgeless graph with vertex set $Y$ whenever $X \subseteq Y$.

- If $Y=\{y\}$, then $\mathcal{B F}_{m}^{*}(X,\{y\})=\mathcal{B F}_{m}(X,\{y\})=\mathcal{B T}_{m}(X \cup\{y\})$.

Define the weighted counts

$$
b f_{m}^{*}(X, Y)=\sum_{H \in \mathcal{B F}_{m}^{*}(X, Y)} w(H) .
$$

Since $\mathcal{B F}_{m}(X, Y) \subseteq \mathcal{B F}_{m}^{*}(X, Y)$, Proposition 8.2 will follow from the stronger result:

Proposition 8.7 For all $X, Y \subseteq V(G)$ with $Y \neq \varnothing$ and all $\alpha \in(1,2]$, we have

$$
\sum_{m=0}^{\infty}\left(\frac{\alpha \Lambda}{\ln \alpha}\right)^{-m} b f_{m}^{*}(X, Y) \leqslant \alpha^{|Y|-1} .
$$


Proof. Let $c=\alpha / \ln \alpha$. We shall show that

$$
\sum_{m=0}^{M}(c \Lambda)^{-m} b f_{m}^{*}(X, Y) \leqslant \alpha^{|Y|-1}
$$

for all $M \geqslant 0$, by using induction on $M+|X|+|Y|$. As noted above, we may assume that $X \cap Y=\varnothing$. The case $M=0$ with $X, Y$ arbitrary holds by the remark after the definition of $\mathcal{B F}_{m}^{*}(X, Y)$. The proposition also holds when $|X \cup Y|=1$ and $M$ is arbitrary, since $b f_{m}^{*}(\varnothing,\{y\})=\delta_{m 0}$. So assume $|X \cup Y| \geqslant 2$ and choose $y \in Y$. By Proposition 3.12, there exists a cocycle $C=E_{G}(L, R)$ in $G$ and a vertex $z \in X \cup Y \backslash y$ such that $z \in L$, $X \cup Y \backslash z \subseteq R$, and $\sum_{e \in C} w_{e} \leqslant \Lambda$. Later we shall distinguish two cases, depending on whether $z$ happens to lie in $X$ or in $Y$.

For $H \in \mathcal{B F}_{m}^{*}(X, Y)$, let $F:=F(H)$ be the set of edges of $H$ that occur as the first edge in $C$ on some path in $H$ from $z$ to $X \cup Y \backslash z$. Let $H_{1}$ be the connected component of $H \backslash F$ containing $z$, and let $H_{2}=H \backslash V\left(H_{1}\right)$. Let $L^{F}$ (resp. $R^{F}$ ) be the set of vertices of $L$ (resp. $R$ ) that are incident with $F$; clearly $\left|L^{F}\right|,\left|R^{F}\right| \leqslant|F|$. (This construction is illustrated in Figure 1.) For each nonempty $F \subseteq C$, let $\mathcal{B F}_{m}^{*}(X, Y ; F)$ be the set of all subgraphs $H \in \mathcal{B F}_{m}^{*}(X, Y)$ such that $F(H)=F$.

Consider now the following two cases:

Case 1: $\boldsymbol{z} \in \boldsymbol{X}$. Then $\mathcal{B F}_{m}^{*}(X, Y ; \varnothing)=\varnothing$. We shall show that, for each nonempty $F \subseteq C$ with $|F|=f$ and each $H \in \mathcal{B F}_{m}(X, Y ; F)$, we have $H_{1} \in \mathcal{B F}_{i}^{*}\left(L^{F},\{z\}\right)=$ $\mathcal{B T}_{i}\left(L^{F} \cup\{z\}\right)$ and $H_{2} \in \mathcal{B F}_{m-f-i}^{*}\left(X \backslash z, Y \cup R^{F}\right)$ for some $i(0 \leqslant i \leqslant m-f)$. Note that by the above definitions $L^{F} \cup\{z\} \subseteq V\left(H_{1}\right)$ and $(X \backslash z) \cup\left(Y \cup R^{F}\right) \subseteq V\left(H_{2}\right)$.

We first consider $H_{1}$, which is the component of $H \backslash F$ containing $z$. Let $B_{1}$ be an end block of $H_{1}$. Then $B_{1}$ is either an end block of $H$, and hence $z \in \operatorname{Int}\left(B_{1}, H_{1}\right)$ or else $B_{1}$ is not an end block of $H$ and hence $\operatorname{Int}\left(B_{1}, H_{1}\right) \cap L^{F} \neq \varnothing$. If $H_{1}$ is non-separable then either $V\left(H_{1}\right)=\{z\}$, or else $H_{1}$ contains $z$ and at least one vertex of $L^{F} \backslash z$ (otherwise $H_{1}$ would be an end block of $H$ with no internal vertex in $X \cup Y)$. Thus $H_{1} \in \mathcal{B F}_{i}^{*}\left(L^{F},\{z\}\right)$ for some $i, 0 \leqslant i \leqslant m-f$.

We next consider $H_{2}=H \backslash V\left(H_{1}\right)$. Each component of $H_{2}$ is either a component of $H$, and hence contains a vertex of $Y$, or is not a component of $H$, and hence contains a vertex of $R^{F}$. Let $B_{2}$ be an end block of $H_{2}$. Then $B_{2}$ is either an end block of $H$, and hence satisfies $\operatorname{Int}\left(B_{2}, H_{2}\right) \cap[(X \backslash z) \cup Y] \neq \varnothing$, or is not an end block of $H$, and hence satisfies $\operatorname{Int}\left(B_{2}, H_{2}\right) \cap R^{F} \neq \varnothing$. Each isolated vertex of $H_{2}$ is either an isolated vertex of $H$, and hence belongs to $Y$, or is not an isolated vertex of $H$, and hence belongs to $R^{F}$. Let $B_{3}$ be an isolated block of $H_{2}$ which is not a single vertex. Then either: $B_{3}$ is an isolated block of $H$, and hence contains at least two vertices of $(X \backslash z) \cup Y$; or $B_{3}$ is an end block of $H$, and hence contains two distinct vertices, one in $(X \backslash z) \cup Y$ and the other in $R^{F}$; or $B_{3}$ is not a block of $H$, and hence contains two distinct vertices of $R^{F}$. Thus $H_{2} \in \mathcal{B F}_{m-f-i}^{*}\left(X \backslash z, Y \cup R^{F}\right)$. 


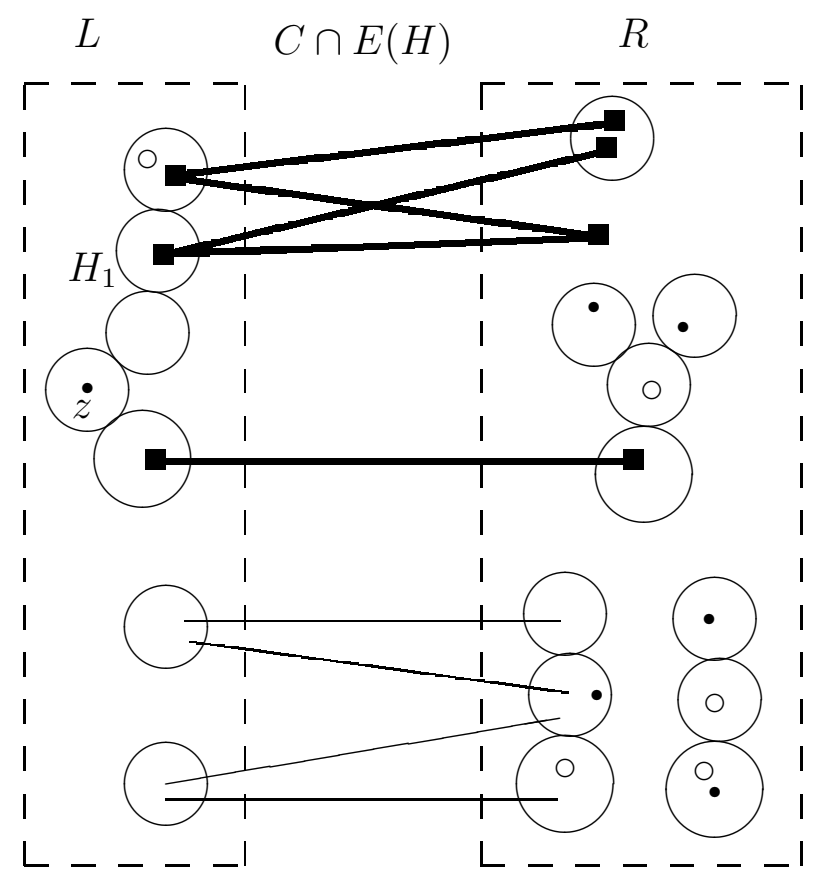

Figure 1: The graph $H \in \mathcal{B F}_{m}^{*}(X, Y)$. Blocks of $H_{1}$ and $H_{2}=H-H_{1}$ are either isolated vertices or else are indicated schematically by large open circles; the edges of $C \cap E(H)$ are shown explicitly, with those in $F$ drawn in bold. Vertices in $X$ are indicated by small solid circles, vertices in $Y$ are indicated by small open circles, and vertices in $L^{F}, R^{F}$ by small solid squares. Note that $H_{2}$ here contains one isolated vertex (which belongs to $R^{F}$ ) and that the four edges in $C \cap E(H)$ that do not belong $F$ (which appear at the bottom of the figure) belong to $H_{2}$ and do not generate vertices of $L^{F}$ or $R^{F}$. 
It follows that the map $H \mapsto\left(H_{1}, H_{2}\right)$ is a weight-preserving injection from $\mathcal{B F}_{m}^{*}(X, Y ; F)$ into $\bigcup_{i=0}^{m-f} \mathcal{B} \mathcal{F}_{i}^{*}\left(L^{F},\{z\}\right) \times \mathcal{B F}_{m-f-i}^{*}\left(X \backslash z, Y \cup R^{F}\right)$. Therefore,

$$
b f_{m}^{*}(X, Y) \leqslant \sum_{\varnothing \neq F \subseteq C} w(F) \sum_{i=0}^{m-f} b f_{i}^{*}\left(L^{F},\{z\}\right) b f_{m-f-i}^{*}\left(X \backslash z, Y \cup R^{F}\right) .
$$

It follows that

$$
\begin{aligned}
& \sum_{m=0}^{M}(c \Lambda)^{-m} b f_{m}^{*}(X, Y) \\
\leqslant & \sum_{m=0}^{M}(c \Lambda)^{-m} \sum_{\varnothing \neq F \subseteq C} w(F) \sum_{i=0}^{m-f} b f_{i}^{*}\left(L^{F},\{z\}\right) b f_{m-f-i}^{*}\left(X \backslash z, Y \cup R^{F}\right) \\
= & \sum_{\varnothing \neq F \subseteq C} w(F)(c \Lambda)^{-f} \sum_{i=0}^{M-f}(c \Lambda)^{-i} b f_{i}^{*}\left(L^{F},\{z\}\right) \sum_{j=0}^{M-f-i}(c \Lambda)^{-j} b f_{j}^{*}\left(X \backslash z, Y \cup R^{F}\right) \\
\leqslant & \sum_{\varnothing \neq F \subseteq C} w(F)(c \Lambda)^{-f} \alpha^{|Y|-1+f}
\end{aligned}
$$

by the inductive hypothesis on $M+|X|+|Y|$. Using Lemma 7.2, we deduce that

$$
\begin{aligned}
\sum_{m=0}^{M}(c \Lambda)^{-m} b f_{m}^{*}(X, Y) & \leqslant \sum_{f=1}^{\infty}(c \Lambda)^{-f} \alpha^{|Y|-1+f} \sum_{\substack{F \subseteq C \\
|F|=f}} w(F) \\
& \leqslant \sum_{f=1}^{\infty}(c \Lambda)^{-f} \alpha^{|Y|-1+f} \frac{\Lambda^{f}}{f !} \\
& =\alpha^{|Y|-1}\left(e^{\alpha / c}-1\right) \leqslant \alpha^{|Y|-1}
\end{aligned}
$$

since $c=\alpha / \ln \alpha$ and $\alpha \leqslant 2$. Thus the proposition holds when $z \in X$.

Case 2: $\boldsymbol{z} \in \boldsymbol{Y}$. Then $y, z \in Y$ and hence $|Y| \geqslant 2$. We can show in a similar way to Case 1 that, for each $F \subseteq C$ with $|F|=f$ and each $H \in \mathcal{B F}_{m}^{*}(X, Y ; F)$, we have $H_{1} \in \mathcal{B F}_{i}^{*}\left(L^{F},\{z\}\right)$ and $H_{2} \in \mathcal{B F}_{m-f-i}^{*}\left(X,(Y \backslash z) \cup R^{F}\right)$ for some $i(0 \leqslant i \leqslant m-f)$. [Let us remark that if $F=\varnothing$, then $H_{1}$ is a component of $H$ consisting of just the isolated vertex z.] The map $H \mapsto\left(H_{1}, H_{2}\right)$ is an injection from $\mathcal{B F}_{m}^{*}(X, Y ; F)$ into $\bigcup_{i=0}^{m-f} \mathcal{B} \mathcal{F}_{i}^{*}\left(L^{F},\{z\}\right) \times \mathcal{B F}_{m-f-i}^{*}\left(X,(Y \backslash z) \cup R^{F}\right)$. Thus

$$
b f_{m}^{*}(X, Y) \leqslant \sum_{F \subseteq C} w(F) \sum_{i=0}^{m-f} b f_{i}^{*}\left(L^{F},\{z\}\right) b f_{m-f-i}^{*}\left(X,(Y \backslash z) \cup R^{F}\right) .
$$


Hence

$$
\begin{aligned}
& \sum_{m=0}^{M}(c \Lambda)^{-m} b f_{m}^{*}(X, Y) \\
\leqslant & \sum_{m=0}^{M}(c \Lambda)^{-m} \sum_{F \subseteq C} w(F) \sum_{i=0}^{m-f} b f_{i}^{*}\left(L^{F},\{z\}\right) b f_{m-f-i}^{*}\left(X,(Y \backslash z) \cup R^{F}\right) \\
= & \sum_{F \subseteq C} w(F)(c \Lambda)^{-f} \sum_{i=0}^{M-f}(c \Lambda)^{-i} b f_{i}^{*}\left(L^{F},\{z\}\right) \sum_{j=0}^{M-f-i}(c \Lambda)^{-j} b f_{j}^{*}\left(X,(Y \backslash z) \cup R^{F}\right) \\
\leqslant & \sum_{F \subseteq C} w(F)(c \Lambda)^{-f} \alpha^{|Y|-2+f}
\end{aligned}
$$

by the inductive hypothesis on $M+|X|+|Y|$. (Note that when $F=\varnothing$, we have $L^{F}=$ $R^{F}=\varnothing$ and hence $\left|(Y \backslash z) \cup R^{F}\right|=|Y|-1$.) Using Lemma 7.2, we deduce that

$$
\begin{aligned}
\sum_{m=0}^{M}(c \Lambda)^{-m} b f_{m}^{*}(X, Y) & \leqslant \sum_{f=0}^{\infty}(c \Lambda)^{-f} \alpha^{|Y|-2+f} \sum_{\substack{F \subseteq C \\
|F|=f}} w(F) \\
& \leqslant \sum_{f=0}^{\infty}(c \Lambda)^{-f} \alpha^{|Y|-2+f} \frac{\Lambda^{f}}{f !} \\
& =\alpha^{|Y|-2} e^{\alpha / c}=\alpha^{|Y|-1}
\end{aligned}
$$

since $c=\alpha / \ln \alpha$. Thus the proposition holds when $z \in Y$.

Remark. The proof technique of Case 1 can be used to prove the induction hypothesis

$$
\sum_{m=0}^{M}(c \Lambda)^{-m} b f_{m}^{*}(X, Y) \leqslant \alpha^{|Y|-1}
$$

whenever $e^{\alpha / c} \leqslant 2$. (In particular, it can handle the apparently best-possible values $c=1 / \ln 2$ and $\alpha=1$.) Likewise, the proof technique of Case 2 can be used to prove the induction hypothesis (8.25) whenever $e^{\alpha / c} \leqslant \alpha$. The trouble is that we need the same hypothesis to work for both cases, since we don't know a priori whether $z$ will lie in $X$ or in $Y$. Therefore, the best we can do - at least with this proof technique - seems to be to choose $\alpha \in(1,2]$ and then set $c=\alpha / \ln \alpha$.

Example 8.4. Let $T$ be any tree (e.g. a long path would do), and let $G$ be the graph $T^{(s)}$ obtained from $T$ by replacing each edge by $s$ parallel edges. Let all edge weights $w_{e}$ 
equal $\Lambda / s$, so that the maxmaxflow is $\Lambda$. Let $x, y \in V\left(T^{(s)}\right)$ with $\operatorname{dist}(x, y)=\ell$. Then $b f_{m}(\{x\},\{y\})=b f_{m}^{*}(\{x\},\{y\})$, and we have

$$
\sum_{m=0}^{\infty} \zeta^{m} b f_{m}(\{x\},\{y\})=\left[\left(1+\frac{\Lambda}{s} \zeta\right)^{s}-1\right]^{\ell} \stackrel{s \rightarrow \infty}{\longrightarrow}\left(e^{\Lambda \zeta}-1\right)^{\ell}
$$

Since $\ell$ can be arbitrarily large, a universal upper bound on $\sum_{m=0}^{\infty} \zeta^{m} b f_{m}(X, Y)$ is impossible for $\zeta>(\ln 2) / \Lambda$, even when $|X|=|Y|=1$.

Example 8.5. Let $G$ be the union of $k$ disjoint copies of $K_{1, r}^{(s)}$, with all edge weights $w_{e}=\Lambda / s$. Let $X$ be the central vertices and $Y$ the remaining vertices, so that $|X|=k$ and $|Y|=k r$. Then

$$
\sum_{m=0}^{\infty} \zeta^{m} b f_{m}(X, Y)=r^{k}\left[\left(1+\frac{\Lambda}{s} \zeta\right)^{s}-1\right]^{k} \stackrel{s \rightarrow \infty}{\longrightarrow} r^{k}\left(e^{\Lambda \zeta}-1\right)^{k}
$$

and

$$
\sum_{m=0}^{\infty} \zeta^{m} b f_{m}^{*}(X, Y)=\left[\left(1+\frac{\Lambda}{s} \zeta\right)^{r s}-1\right]^{k} \stackrel{s \rightarrow \infty}{\longrightarrow}\left(e^{r \Lambda \zeta}-1\right)^{k} .
$$

Thus, if any universal upper bound is possible for $\zeta=(\ln 2) / \Lambda$, the right-hand side has to be at least $(|Y| /|X|)^{|X|}$ for $b f$ and $\left(2^{|Y| /|X|}-1\right)^{|X|}$ for $b f^{*}$.

These examples suggest the following conjectures:

Conjecture 8.8 For all $X, Y \subseteq V(G)$ with $Y \neq \varnothing$, we have

$$
\sum_{m=0}^{\infty}\left(\frac{\Lambda}{\ln 2}\right)^{-m} b f_{m}(X, Y) \leqslant|Y|^{|X|}
$$

Conjecture 8.9 For all $X, Y \subseteq V(G)$ with $Y \neq \varnothing$, we have

$$
\sum_{m=0}^{\infty}\left(\frac{\Lambda}{\ln 2}\right)^{-m} b f_{m}^{*}(X, Y) \leqslant 2^{|Y|}-1
$$

Conjecture 8.10 (a special case of Conjecture 8.8 or 8.9) For all nonempty $X \subseteq$ $V(G)$, we have

$$
\sum_{m=0}^{\infty}\left(\frac{\Lambda}{\ln 2}\right)^{-m} b t_{m}(X) \leqslant 1
$$




\subsection{The class $\mathcal{B}_{m}(X)$}

We conclude by discussing a larger class of block forests, which is roughly the block analogue of the class $\mathcal{H}_{m}(X)$ considered in Section 6.2. For $X \subseteq V(G)$, let $\mathcal{B}_{m}(X)$ be the set of all $m$-edge subgraphs $H$ in $G$ (connected or not) such that

(B1) $X \subseteq V(H)$;

(B2) each end block of $H$ contains at least one element of $X$ as an internal vertex [that is, $\operatorname{Int}(B, H) \cap X \neq \varnothing]$; and

(B3) each isolated block of $H$ is either an isolated vertex belonging to $X$ or else contains at least two vertices of $X$.

[It follows from (B2) and (B3) that each component of $H$ is either an isolated vertex belonging to $X$ or else contains at least two vertices of $X$. In particular, $\mathcal{B}_{m}(X) \subseteq \mathcal{C}_{m}(X)$. Note also that $\mathcal{B F}_{m}(X, Y) \subseteq \mathcal{B F}_{m}^{*}(X, Y) \subseteq \mathcal{B}_{m}(X \cup Y)$.] Note the following special cases:

- For any $X, \mathcal{B}_{0}(X)$ has as its single element the edgeless graph with vertex set $X$.

- $\mathcal{B}_{m}(\varnothing)=\varnothing$ for $m \geqslant 1$.

- $\mathcal{B}_{m}(\{x\})=\varnothing$ for $m \geqslant 1$.

- $\mathcal{B}_{m}(\{x, y\})$ is the set of $m$-edge $x y$-block paths when $x \neq y$ and $m \geqslant 1$. [Hence $\mathcal{B}_{m}(\{x, y\})=\mathcal{B T}_{m}(\{x, y\})=\mathcal{B F}_{m}(\{x\},\{y\})=\mathcal{B F}_{m}^{*}(\{x\},\{y\})$.]

Recall that $w(H)=\prod_{e \in E(H)} w_{e}$. Define the weighted counts

$$
b_{m}(X)=\sum_{H \in \mathcal{B}_{m}(X)} w(H) .
$$

We then have the following bound in terms of maxmaxflow:

Proposition 8.11 Whenever $|X|=k \geqslant 1$ we have

$$
b_{m}(X) \leqslant B(m, k) \Lambda^{m}
$$

where

$$
B(m, k)=2^{m} C\left(m, \frac{k-1}{2}\right)= \begin{cases}(k-1)(2 m+k-1)^{m-1} / m ! & \text { for } k \neq 1 \\ \delta_{m 0} & \text { for } k=1\end{cases}
$$

For the case of greatest interest, namely $\mathcal{B}_{m}(\{x, y\})=\mathcal{B F}_{m}^{*}(\{x\},\{y\})$, the bound of Proposition 8.11 behaves roughly like $(2 e \Lambda)^{m}$, which is much worse than the bound $(2 \Lambda / \ln 2)^{m}$ of Propositions 8.2 and 8.7. However, as we shall see, the proof of Proposition 8.11 is quite a bit simpler than that of Proposition 8.7.

Before beginning the proof of Proposition 8.11, let us note some facts about the numbers $B(m, k)$, which follow easily from the facts about the $C(m, k)$ already discussed in Section 7: 
(a) For each integer $m \geqslant 0, B(m, k)$ is an increasing function of $k \geqslant 1$.

(b) Generating function: If $\mathrm{C}(z)$ solves the equation (7.4), then

$$
\mathrm{C}(2 z)^{(k-1) / 2}=\sum_{m=0}^{\infty} B(m, k) z^{m}
$$

This is an immediate translation of (7.5).

(c) For all $k_{1}, k_{2}, m$ we have

$$
\sum_{i=0}^{m} B\left(i, k_{1}\right) B\left(m-i, k_{2}\right)=B\left(m, k_{1}+k_{2}-1\right) .
$$

This is an immediate consequence of (8.35).

(d) For all $k$,

$$
B(m, k)=\sum_{f=0}^{m} \frac{1}{f !} B(m-f, k-1+2 f) .
$$

This is an immediate translation of the identity (7.7) with $z=1 / 2$.

(e) For each fixed $k>1$, we have

$$
B(m, k)=(2 e)^{m} m^{-3 / 2} \frac{(k-1) e^{(k-1) / 2}}{\sqrt{8 \pi}}[1+O(1 / m)]
$$

as $m \rightarrow \infty$. This is an immediate translation of (7.9).

Proof of Proposition 8.11. The Proposition holds trivially when $k=1$, so we may assume that $k \geqslant 2$. Choose $x_{1}, x_{2} \in X$ and let $C$ be a minimum cut in $G$ separating $x_{1}$ from $x_{2}$, so that $\sum_{e \in C} w_{e} \leqslant \Lambda$. Let $G_{1}$ be the component of $G-C$ containing $x_{1}$, and let $G_{2}$ be the union of the remaining components of $G-C$. By construction, we have $x_{1} \in V\left(G_{1}\right)$ and $x_{2} \in V\left(G_{2}\right)$, and each edge in $C$ joins a vertex of $G_{1}$ to a vertex of $G_{2}$. Let $X_{i}=X \cap V\left(G_{i}\right)$ and $k_{i}=\left|X_{i}\right|$ for $i=1,2$. Since $x_{1} \in X_{1}$ and $x_{2} \in X_{2}$, we have $1 \leqslant k_{i} \leqslant k-1$ for $i=1,2$; and of course $k_{1}+k_{2}=k$.

We shall classify the subgraphs $H$ of $G$ belonging to $\mathcal{B}_{m}(X)$ according to $F \equiv E(H) \cap$ $C$. So, for each $F \subseteq C$, let us set $f=|F|$; define $Y_{i}^{F}(i=1,2)$ to be the set of vertices of $G_{i}$ that are incident with $F$ (note that $\left|Y_{i}^{F}\right| \leqslant f$ ); and finally, let $\mathcal{B}_{m}(X ; F)$ be the set of all $H \in \mathcal{B}_{m}(X)$ that have $E(H) \cap C=F$.

For $H \in \mathcal{B}_{m}(X ; F)$, we let $H_{i}=H \cap G_{i}$ and $m_{i}=\left|E\left(H_{i}\right)\right|$; note that $H=H_{1} \cup H_{2} \cup F$ and $m=m_{1}+m_{2}+f$. We shall show that $H_{i} \in \mathcal{B}_{m_{i}}\left(X_{i} \cup Y_{i}^{F}\right)$ for $i=1,2$. (The argument is illustrated in Figure 2.) Note first that since $X \subseteq V(H)$ we have $X_{i} \subseteq$ $V\left(H_{i}\right)$. Furthermore, each end block $B$ of $H_{i}$ is either an end block of $H$ or else satisfies 


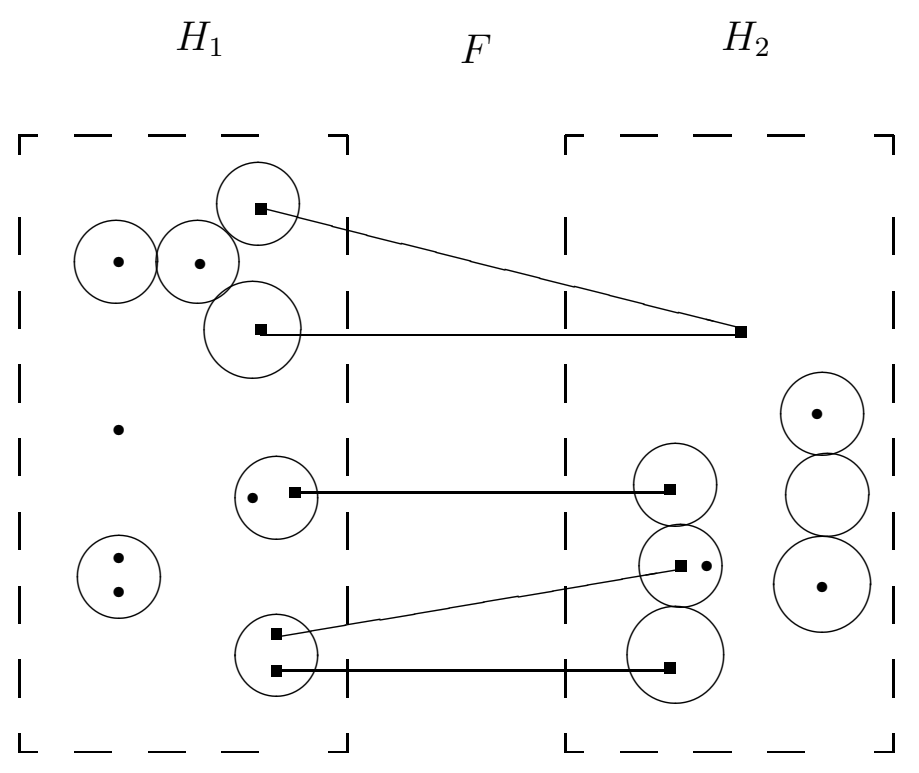

Figure 2: The graph $H \in \mathcal{B}_{m}(X)$. Blocks of $H_{1}$ and $H_{2}$ are indicated schematically by large open circles; the edges of $F$ are shown explicitly. Vertices in $X$ are indicated by small solid circles, and vertices in $Y_{1}^{F}, Y_{2}^{F}$ are indicated by small solid squares.

$\operatorname{Int}\left(B, H_{i}\right) \cap Y_{i}^{F} \neq \varnothing$. Hence $\operatorname{Int}\left(B, H_{i}\right) \cap\left(X_{i} \cup Y_{i}^{F}\right) \neq \varnothing$. Each isolated vertex $v$ of $H_{i}$ is either an isolated vertex of $H$ or else belongs to $Y_{i}^{F}$. Thus $v \in X_{i} \cup Y_{i}^{F}$. Finally, each isolated block $B$ of $H_{i}$ that is not an isolated vertex of $H_{i}$ is either an isolated block of $H$, or else an end block of $H$ with its cut vertex in $Y_{i}^{F}$, or else satisfies $\left|V(B) \cap Y_{i}^{F}\right| \geqslant 2$. Thus $\left|V(B) \cap\left(X_{i} \cup Y_{i}^{F}\right)\right| \geqslant 2$.

It follows that we can construct a weight-preserving [except for a factor $w(F)$ ] bijection of $\mathcal{B}_{m}(X ; F)$ onto a subset of $\bigcup_{i=0}^{m-f} \mathcal{B}_{i}\left(X_{1} \cup Y_{1}^{F}\right) \times \mathcal{B}_{m-f-i}\left(X_{2} \cup Y_{2}^{F}\right)$. Thus

$$
b_{m}(X) \leqslant \sum_{F \subseteq C} w(F) \sum_{i=0}^{m-f} b_{i}\left(X_{1} \cup Y_{1}^{F}\right) b_{m-f-i}\left(X_{2} \cup Y_{2}^{F}\right) .
$$

Since $i \leqslant m-f,\left|Y_{1}^{F}\right| \leqslant f$ and $\left|X_{1}\right|<k$, we have $i+\left|X_{1} \cup Y_{1}^{F}\right|<m+k$; and likewise we have $(m-f-i)+\left|X_{2} \cup Y_{2}^{F}\right|<m+k$. Therefore, we can use induction on $m+k$ (the Proposition being true for the initial case $m+k=1$ ) to deduce that

$$
\begin{aligned}
b_{m}(X) & \leqslant \sum_{F \subseteq C} w(F) \sum_{i=0}^{m-f} \Lambda^{m-f} B\left(i,\left|X_{1} \cup Y_{1}^{F}\right|\right) B\left(m-f-i,\left|X_{2} \cup Y_{2}^{F}\right|\right) \\
& \leqslant \sum_{F \subseteq C} w(F) \sum_{i=0}^{m-f} \Lambda^{m-f} B\left(i, k_{1}+f\right) B\left(m-f-i, k_{2}+f\right)
\end{aligned}
$$




$$
\begin{aligned}
& \leqslant \sum_{f=0}^{m} \frac{\Lambda^{f}}{f !} \sum_{i=0}^{m-f} \Lambda^{m-f} B\left(i, k_{1}+f\right) B\left(m-f-i, k_{2}+f\right) \\
& =\Lambda^{m} B(m, k),
\end{aligned}
$$

where the second line used the fact that $B(m, k)$ increases with $k$ for $k \geqslant 1$, the third line used Lemma 7.2, and the last line used identities (8.36) and (8.37) and the fact that $k_{1}+k_{2}=k$.

Corollary 8.12 Fix a weighted graph $(G, \mathbf{w})$ and an edge $e \in E(G)$. Then the sum of the weights of the non-separable m-edge subgraphs of $G$ containing $e$ is at most $B(m-$ 1,2) $\Lambda(G-e, \mathbf{w})^{m-1} w_{e}$.

Proof. Let $e=x y$ and put $X=\{x, y\}$. Let $H$ be a subgraph of $G$ and let $m \geqslant 1$. Then $H$ is a non-separable $m$-edge subgraph of $G$ containing $e$ if and only if $H-e \in \mathcal{B}_{m-1}(X)$. Thus Proposition 8.11 applied to $G-e$ gives the claimed result.

\section{Acknowledgments}

We wish to thank Jan van den Heuvel and Akira Saito for valuable conversations. We also thank Gordon Royle for drawing our attention to references [1, 9], and for many valuable conversations. Finally, we thank an anonymous referee for several helpful comments, including catching an error in our original versions of Corollaries 8.5 and 8.12 (which were called Corollaries 7.5 and 7.12 in the preprint version of this paper).

This research was supported in part by U.S. National Science Foundation grants PHY-9900769, PHY-0099393 and PHY-0424082. It was begun while one of the authors (A.D.S.) was a Visiting Fellow at All Souls College, Oxford, where he was hosted by the Department of Theoretical Physics and supported in part by U.K. Engineering and Physical Sciences Research Council grant GR/M 71626.

\section{References}

[1] Bollobás, B. (1966) On graphs with at most three independent paths connecting any two vertices. Studia Sci. Math. Hungar. 1, 137-140.

[2] Bollobás, B. (1978) Extremal Graph Theory. Academic Press, London.

[3] Bollobás, B. (1998) Modern Graph Theory. Springer-Verlag, New York.

[4] Gomory, R.E. and Hu, T.C. (1961) Multi-terminal network flows. J. Soc. Indust. Appl. Math. 9, 551-570. Reprinted in D.R. Fulkerson (editor), Studies in Graph Theory, Part I, Studies in Mathematics \#11, pp. 172-199 (Mathematical Association of America, Washington, 1975). 
[5] Jackson, B. (2003) Zeros of chromatic and flow polynomials of graphs, J. Geom. 76, 95-109, math.CO/0205047 at arXiv.org.

[6] Leonard, J.L. (1972) On graphs with at most four line-disjoint paths connecting any two vertices. J. Combin. Theory B 13, 242-250.

[7] Leonard, J.L. (1973) Graphs with 6-ways. Canad. J. Math. 25, 687-692.

[8] Lovász, L. and Plummer, M.D. (1986) Matching Theory. North-Holland Mathematics Studies \#121. Annals of Discrete Mathematics \#29. North-Holland, Amsterdam-New York / Akadémiai Kiadó, Budapest.

[9] Mader, W. (1973) Ein Extremalproblem des Zusammenhangs von Graphen. Math. Z. 131, 223-231.

[10] Mader, W. (1979) Connectivity and edge-connectivity in finite graphs. In B. Bollobás (editor), Surveys in Combinatorics (Proceedings of the Seventh British Combinatorial Conference), London Mathematical Society Lecture Note Series \#38, pp. 66-95. Cambridge University Press, Cambridge.

[11] Royle, G.F. and Sokal, A.D. (2009) Linear bound in terms of maxmaxflow for the chromatic roots of series-parallel graphs, in preparation.

[12] Shrock, R. and Tsai, S.-H. (1998) Ground state degeneracy of Potts antiferromagnets: Cases with noncompact $W$ boundaries having multiple points at $1 / q=0$. J. Phys. A 31, 9641-9665, cond-mat/9810057 at arXiv.org.

[13] Shrock, R. and Tsai, S.-H. (1999) Ground state degeneracy of Potts antiferromagnets: Homeomorphic classes with noncompact $W$ boundaries. Physica A 265, 186-223, cond-mat/9811410 at arXiv.org.

[14] Sokal, A.D. (2001) Bounds on the complex zeros of (di)chromatic polynomials and Potts-model partition functions. Combin. Probab. Comput. 10, 41-77, condmat/9904146 at arXiv.org.

[15] Sokal, A.D. (2004) Chromatic roots are dense in the whole complex plane. Combin. Probab. Comput. 13, 221-261, cond-mat/0012369 at arXiv.org.

[16] Stanley, R.P. (1999) Enumerative Combinatorics, vol. 2. Cambridge University Press, Cambridge-New York.

[17] Thomassen, C. (1997) The zero-free intervals for chromatic polynomials of graphs. Combin. Probab. Comput. 6, 497-506. 